\title{
SESQUITERPENOS PRODUZIDOS PELO FUNGO ENDOFÍTICO Phomopsis cassiae COM ATIVIDADE ANTIFÚNGICA E INIBIDORA DE ACETILCOLINESTERASE ${ }^{\#}$
}

Lisinéia M. Zanardi, Vanderlan da S. Bolzani, Alberto J. Cavalheiro, Dulce H. Siqueira Silva, Henrique C. Trevisan ${ }^{\dagger}$ e Angela R. Araujo*

Instituto de Química, Universidade Estadual Paulista, CP 355, 14801-970 Araraquara - SP, Brasil

Geraldo H. Silva

Centro de Ciências Exatas e Tecnologia, Universidade Federal de Sergipe, 49100-000 Aracajú - SE, Brasil

Helder L. Teles

Departamento de Ciências Biológicas, Universidade Federal do Mato Grosso, 78735-901 Rondonópolis - MT, Brasil

Maria Cláudia M. Young

Secção de Fisiologia e Bioquímica de Plantas, Instituto de Botânica, CP 4005, 01061-970 São Paulo - SP, Brasil

Recebido em 24/4/12; aceito em 24/8/12; publicado na web em 28/9/12

\begin{abstract}
SESQUITERPENES PRODUCED BY ENDOPHYTIC FUNGUS Phomopsis cassiae WITH ANTIFUNGAL AND ACETYLCHOLINESTERASE INHIBITION ACTIVITIES. Two new diastereoisomeric cadinanes sesquiterpenes 3,9-dihydroxycalamenene (1-2), along with the known 3-hydroxycalamen-8-one (3) and aristelegone-A (4), were isolated from ethyl acetate extract of Phomopsis cassiae, an endophytic fungus in Cassia spectabilis. Their structures, including relative stereochemistry, were determined on the basis of detailed interpretation of 2D NMR spectra and comparison with related known compounds. Compounds 1-4 displayed antifungal activity against the phytopathogenic fungi Cladosporium cladosporioides and $C$. sphaerospermum, as well as inhibition of acetylcholinesterase.
\end{abstract}

Keywords: Phomopsis cassia; endophytic fungus; sesquiterpenes.

\section{INTRODUÇÃO}

Os micro-organismos representam uma interessante fonte de produtos naturais estruturalmente diversos e farmacologicamente ativos, com vasta aplicação como agroquímicos, antibióticos, imunossupressores, antiparasíticos e agentes anticancerígenos. ${ }^{1-6}$ Entre estes encontram-se os fungos endofíticos, que são micro-organismos que residem nos espaços inter e intracelulares de plantas vasculares durante um período do seu ciclo de vida, não causando efeitos patogênicos ao hospedeiro. ${ }^{1-6}$ Por meio dessa associação simbiótica, a planta hospedeira protege e nutre o endófito. Este, por sua vez, produz metabólitos que favorecem o crescimento e melhoram a competitividade da planta, além de protegê-la de herbívoros e fitopatógenos. ${ }^{1-6}$ Apesar da importância deste nicho de micro-organismos na produção de metabólitos secundários bioativos e novos, este é ainda muito pouco explorado, sobretudo quando em associações com espécies vegetais brasileiras.

Em continuidade ao projeto de "Prospecção Química e Biológica em Fungos Endofíticos Isolados de Espécies Vegetais de Cerrado", ${ }^{7-11}$ o fungo endofítico Phomopsis cassiae, que se mostrou um excelente produtor de metabólitos novos e bioativos quando cultivado em meio líquido de dextrose batata (PDB), ${ }^{7,10}$ foi selecionado para estudo químico em extrato de malte (EM). O interesse no estudo da produção metabólica de $P$. cassiae em extrato de malte foi avaliar a produção metabólica em diferentes condições nutricionais, como observado em trabalho anterior. ${ }^{9}$

O estudo químico do extrato bruto AcOEt produzido pelo fungo endofítico $P$. cassiae resultou no isolamento de quatro sesquiterpenos da classe dos cadinanos, sendo dois novos estereoisômeros

*e-mail: araujoar@iq.unesp.br

†n Memoriam

\#Artigo em homenagem ao Prof. Otto R. Gottlieb (31/8/1920-19/6/2011)
Rel. (7R,9S,10R)-3,9-di-hidroxicalameneno (1) e Rel. (7R,9R,10R)3,9-di-hidroxicalameneno (2) e os conhecidos Rel. (7S, 10R)-3hidroxicalamen-8-ona (3) e aristelegona-A (4).

As estruturas das substâncias foram determinadas ou elucidadas pela análise dos espectros de infravermelho, EM, RMN de ${ }^{1} \mathrm{H} \mathrm{e}{ }^{13} \mathrm{C}$, uni e bidimensionais.

As substâncias 1-4 foram avaliadas quanto ao potencial antifúngico e inibidor de acetilcolinesterase.

\section{PARTE EXPERIMENTAL}

\section{Procedimentos gerais}

Os espectros de absorção na região do Infravermelho foram registrados em espectrofotômetro da Nicolet, modelo IMPACT-400, em pastilhas de $\mathrm{KBr}$. Os espectros de $\mathrm{RMN}$ de ${ }^{1} \mathrm{H}$ e ${ }^{13} \mathrm{C}$ e experimentos bidimensionais foram realizados em espectrômetro da Varian, modelo Inova-500 (11,7 Tesla) operando na frequência de $500 \mathrm{MHz}$ para o núcleo de ${ }^{1} \mathrm{H}$ e $125 \mathrm{MHz}$ para ${ }^{13} \mathrm{C}$; TMS foi utilizado como referência interna. Os espectros de massas de alta e baixa resolução foram registrados em espectrômetro da Micromass, modelo triplo quadrupolo (Quattro-LC, Manchester, UK). As amostras foram introduzidas utilizando uma seringa de fluxo $\left(10 \mu \mathrm{L} \mathrm{min}^{-1}\right)$.

As análises e separações cromatográficas por CLAE (cromatografia líquida de alta eficiência) foram realizadas em equipamento da Varian, modelo Prostar, acoplado ao detector ProStar 330 de arranjos de diodos, utilizando coluna analítica Phenomenex Fenil-Hexil (250 x 4,60 mm; $5 \mu \mathrm{m})$ e coluna Phenomenex Fenil-Hexil $(250$ x 21,20 $\mathrm{mm} ; 10 \mu \mathrm{m})$, em modo preparativo. Nas separações cromatográficas em coluna aberta sob pressão foi utilizada sílica gel C-18 (Merck Lichroprep $\left.{ }^{\oplus} \mathrm{FR}-18,25-40 \mu \mathrm{m}\right)$ em coluna $20 \times 3,7 \mathrm{~cm}$.

$\mathrm{O}$ cultivo de $P$. cassiae em meio líquido em escala ampliada foi conduzido em 28 frascos de Erlenmeyer $(500 \mathrm{~mL}$ ) contendo 6,0 g de 
EM (extrato de malte) (DIFCO), e $300 \mathrm{~mL}$ de água ultrapura (Mili-Q), que foram esterilizados em autoclave a $121{ }^{\circ} \mathrm{C}$ e 1 atm por $40 \mathrm{~min}$.

\section{Material vegetal}

Folhas de C. spectabilis foram coletadas em junho de 2001, em Araraquara-SP. A identificação botânica foi realizada pela Dra. M. C. M. Young e uma exsicata (SILVA-193) encontra-se depositada no Herbário do Instituto de Botânica da Secretaria do Meio Ambiente, São Paulo, Brasil.

\section{Isolamento da cepa fúngica}

O fungo endofítico P. cassiae foi isolado de acordo com procedimento previamente descrito. ${ }^{7,10}$

\section{Cultivo do micro-organismo e obtenção do extrato bruto}

O fungo endofítico P. cassiae ${ }^{10}$ foi identificado pelo Prof. Dr. L. H. Pfenning e depositado na Coleção Micológica da Universidade Federal de Lavras como CML-292.

P. cassiae foi repicado para placas de Petri contendo batata, dextrose e ágar (PDA) e incubado por 8 dias à temperatura ambiente. $\mathrm{A}$ seguir, foi inoculado em meio de cultivo EM, perfazendo um total de $8,4 \mathrm{~L}$, e mantido em incubadora rotatória a $150 \mathrm{rpm}$ e $25^{\circ} \mathrm{C}$ por 28 dias. Após este período, o caldo foi separado do micélio por filtração e particionado com AcOEt ( 3 x 4,2 L). A fase orgânica foi seca com $\mathrm{MgSO}_{4}$ e concentrada, fornecendo $658,1 \mathrm{mg}$ de extrato bruto.

\section{Extração e isolamento dos constituintes químicos}

O extrato bruto $(658,1 \mathrm{mg})$ foi fracionado em coluna cromatográfica $(20 \times 3,7 \mathrm{~cm})$ usando-se sílica gel de fase reversa C-18. Primeiramente eluída com gradiente de $\mathrm{H}_{2} \mathrm{O}-\mathrm{MeOH}(90: 10 \rightarrow 100 \%$ $\mathrm{MeOH}$ ), forneceu 14 frações; a seguir, com MeOH:AcOEt 50:50 e AcOEt puro, forneceu mais 2 frações. Após criteriosa análise por CCDC (cromatografia em camada delgada comparativa) de sílica gel, usando-se Hex:AcOEt (3:97) e AcOEt:MeOH (98:2) como eluente, as frações Pc-EM-07 (49,0 mg) e Pc-EM-09 (55,0 mg) foram selecionadas para fracionamento cromatográfico por $\mathrm{CLAE}_{\text {prep. }}$ (cromatografia liquida de alta eficiência preparativa). A fração Pc-EM-09 foi submetida à CLAE ${ }_{\text {prep. }}$ em fase reversa $\left[\lambda=220 \mathrm{~nm} ; 12,0 \mathrm{~mL} \mathrm{~min}^{-1}\right.$; $\left.\mathrm{CH}_{3} \mathrm{CN}: \mathrm{H}_{2} \mathrm{O}(50: 50 \rightarrow 30: 70), 25 \mathrm{~min}\right]$, fornecendo $\mathbf{1}\left(3,1 \mathrm{mg}, \mathrm{t}_{\mathrm{R}}=\right.$ $13,5 \mathrm{~min}), 2\left(2,0 \mathrm{mg}, \mathrm{t}_{\mathrm{R}}=13,0 \mathrm{~min}\right)$ e $3\left(2,3 \mathrm{mg}, \mathrm{t}_{\mathrm{R}}=16,5\right)$. A fração Pc-EM-07 foi submetida à CLAE prep. $_{\text {em fase reversa }}[\lambda=254 \mathrm{~nm} ; 15$ $\left.\mathrm{mL} \mathrm{min}{ }^{-1} ; \mathrm{CH}_{3} \mathrm{CN}: \mathrm{H}_{2} \mathrm{O}(35: 65)\right]$, fornecendo 4 (2,3 mg, $\left.\mathrm{t}_{\mathrm{R}}=18 \mathrm{~min}\right)$.

\section{Bioensaio}

Ensaio para avaliação da atividade antifúngica

Para avaliação da atividade antifúngica do extrato bruto AcOEt e das substâncias 1-4 contra os fungos fitopatogênicos C. cladosporioides e $C$. sphaerospermum foi utilizada a metodologia descrita anteriormente. ${ }^{12}$ Os ensaios foram realizados nas concentrações de $1,5,10,25,50$ e $100 \mu \mathrm{g} \mathrm{mL}^{-1}$, usando nistatina $\left(1 \mu \mathrm{g} \mathrm{mL}{ }^{-1}\right)$ como controle positivo.

\section{Ensaio de inibição da enzima acetilcolinesterase por CCDC}

Para avaliação da atividade anticolinesterásica das substâncias 1-4 foi utilizado o método colorimétrico de Ellman em cromatografia em camada delgada. ${ }^{13}$ Os ensaios foram realizados nas concentrações de $1,3,10,30$ e $60 \mu \mathrm{M}$ das substâncias puras, usando galantamina $(1 \mu \mathrm{M})$ como controle positivo.
Rel. (7R,9S,10R)-3,9-di-hidroxicalameneno (1)

Óleo amarelo claro $(3,1 \mathrm{mg}) ;[\alpha]_{\mathrm{D}}^{25}-79,6(c$ 0,25, MeOH); IV $(\mathrm{KBr}) \mathrm{cm}^{-1}: 3442,1633,1460$. HRESI-MS: $\mathrm{m} / z$ 257,1511 [M+Na $]^{+}$, $m / z, 217,1602\left[\mathrm{M}-\mathrm{H}_{2} \mathrm{O}+\mathrm{H}\right]^{+}$. Calcd. para $\mathrm{C}_{15} \mathrm{H}_{22} \mathrm{O}_{2} 217,1592$. RMN de ${ }^{1} \mathrm{H}\left(500 \mathrm{MHz}, \mathrm{CDCl}_{3}\right) \delta: 2,65(q, J=6,5 \mathrm{~Hz}, \mathrm{H}-10), 3,88(q, J$ $=6,5$ e $5,5 \mathrm{~Hz}, \mathrm{H}-9), 1,72(d d d, J=13,0 ; 7,0$ e $6,5 \mathrm{~Hz}, \mathrm{H}-8), 1,75$ ( $d d d, J=13,0 ; 7,0$ e 3,0 Hz, H-8), 2,72 ( $m, \mathrm{H}-7), 6,91$ ( $s, \mathrm{H}-5), 6,48$ $(s, \mathrm{H}-2), 2,20$ ( $m, \mathrm{H}-11), 0,67$ ( $d, J=6,5 \mathrm{~Hz}, \mathrm{H}-12), 0,95(d, J=7,0$ $\mathrm{Hz}, \mathrm{H}-13), 1,18(d, J=7,0 \mathrm{~Hz}, \mathrm{H}-14), 2,13$ ( $s, \mathrm{H}-15)$. RMN de ${ }^{13} \mathrm{C}$ (125 MHz, $\mathrm{CDCl}_{3}$ ) $\delta: 41,5$ (C-10), 71,2 (C-9), 26,9 (C-8), 39,0 (C-7), 130,4 (C-6), 130,2 (C-5), 39,0 (C-7), 152,0 (C-3), 115,1 (C-2), 138,6 (C-1), 31,3 (C-11), 17,2 (C-12), 21,1 (C-13), 21,4 (C-14), 15,5 (C-15).

\section{Rel. (7R,9R,10R)-3,9-di-hidroxicalameneno (2)}

Óleo amarelo claro $(2,0 \mathrm{mg}) ;[\alpha]_{\mathrm{D}}^{25}-80,5$ (c 0,25, MeOH); IV $(\mathrm{KBr}) \mathrm{cm}^{-1}: 3440,1625,1460$. HRESI-MS : $\mathrm{m} / z$ 257,1509 [M+Na $]^{+}$, $m / z, 273,1239[\mathrm{M}+\mathrm{K}]^{+}, m / z, 217,1593\left[\mathrm{M}-\mathrm{H}_{2} \mathrm{O}+\mathrm{H}\right]^{+}$, Calcd. para $\mathrm{C}_{15} \mathrm{H}_{22} \mathrm{O}_{2} 217,15924$. RMN de ${ }^{1} \mathrm{H}\left(500 \mathrm{MHz}, \mathrm{CDCl}_{3}\right) \delta: 2,83(\mathrm{~m}$, $\mathrm{H}-10), 3,99$ ( $d d d, J=12,0 ; 4,5 ; 4,5 \mathrm{~Hz}, \mathrm{H}-9), 1,58$ ( $m, \mathrm{H}-8), 1,70$ ( $m, \mathrm{H}-8), 2,80$ ( $m, \mathrm{H}-7), 6,93$ ( $s, \mathrm{H}-5), 6,43$ ( $s, \mathrm{H}-2), 2,35$ ( $m, \mathrm{H}-11)$, $0,98(d, J=7,0 \mathrm{~Hz}, \mathrm{H}-12), 0,59(d, J=7,0 \mathrm{~Hz}, \mathrm{H}-13), 1,11(d, J=7,0$ $\mathrm{Hz}, \mathrm{H}-14), 2,13$ (s, H-15). RMN de ${ }^{13} \mathrm{C}\left(125 \mathrm{MHz} \mathrm{CDCl}_{3}\right) \delta: 39,9$ (C-10), 70,2 (C-9), 26,0 (C-8), 42,5 (C-7), 130,3 (C-6), 129,5 (C-5), 122,2 (C-4), 151,9 (C-3), 115,2 (C-2), 141,4 (C-1), 31,1 (C-11), 21,0 (C-12), 16,4 (C-13), 15,9 (C-14), 15,9 (C-15).

\section{Rel. (7S,10R)-3-hidroxicalamen-8-ona (3)}

Óleo amarelo claro $(2,3 \mathrm{mg}) ;[\alpha]_{\mathrm{D}}^{25}+9,9$ ( $\left.0.25, \mathrm{MeOH}\right)$; IV $(\mathrm{KBr}) \mathrm{cm}^{-1}: 3448,1635,1460$. HRESI-MS: $m / z$ 233,1542 [M+H] $]^{+}$, $m / z, 255,1452[\mathrm{M}+\mathrm{Na}]^{+}, m / z, 271,1201[\mathrm{M}+\mathrm{K}]^{+}$, Calcd. para $\mathrm{C}_{15} \mathrm{H}_{20} \mathrm{O}_{2}$ 233,15423. RMN de ${ }^{1} \mathrm{H}\left(500 \mathrm{MHz}, \mathrm{CDCl}_{3}\right) \delta: 3,27$ (m, H-10), 2,62 $\left(d d, J=17,5 ; 5,5 \mathrm{~Hz}, \mathrm{H}-9_{\mathrm{A}}\right), 2,05\left(\mathrm{dd}, \mathrm{J}=17,5 ; 11,5 \mathrm{~Hz}, \mathrm{H}-9_{\mathrm{B}}\right), 2,91$ (d, $J=8,0 ; \mathrm{Hz}, \mathrm{H}-7), 6,74$ ( $s, \mathrm{H}-5), 6,67$ ( $s, \mathrm{H}-2), 2,13$ ( $m, \mathrm{H}-11$ ), $0,91(d, J=7,0 \mathrm{~Hz}, \mathrm{H}-12), 0,78(d, J=6,5 \mathrm{~Hz}, \mathrm{H}-13), 1,24(d, J=$ $7,0 \mathrm{~Hz}, \mathrm{H}-14), 2,12(s, \mathrm{H}-15)$. RMN de ${ }^{13} \mathrm{C}\left(125 \mathrm{MHz}, \mathrm{CDCl}_{3}\right) \delta$ : 31,0 (C-10), 213,0 (C-8), 62,0 (C-7), 133,0 (C-5), 123,0(C-4), 153,0 (C-3), 113,0 (C-2), 140,0 (C-10), 32,0 (C-11), 15,1 (C-12), 19,0 (C-13), 21,0 (C-14), 15,5 (C-15).

\section{Aristelegona-A (4)}

Óleo amarelo claro $(2,3 \mathrm{mg}) ;[\alpha]_{\mathrm{D}}^{25}+32,6(c 0.25, \mathrm{MeOH}) ; \mathrm{IR}$ $(\mathrm{KBr}) \mathrm{cm}^{-1}: 3458,1652,1600$. HRESI-MS: $m / z$ 191,1131 $[\mathrm{M}+\mathrm{H}]^{+}$, $m / z, 213,0954[\mathrm{M}+\mathrm{Na}]^{+}$. Calcd. para $\mathrm{C}_{12} \mathrm{H}_{14} \mathrm{O}_{2} 191,1073$. RMN de ${ }^{1} \mathrm{H}$ $\left(500 \mathrm{MHz}, \mathrm{CDCl}_{3}\right) \delta: 2,47\left(d d d, J=17,0 ; 8,5 ; 4,5 \mathrm{~Hz}, \mathrm{H}-2_{\mathrm{A}}\right), 2,65$ $\left(d d d, J=17,0 ; 8,0 ; 4,5 \mathrm{~Hz}, \mathrm{H}-2_{\mathrm{B}}\right), 1,79$ (dddd, $J=13,0 ; 8,5 ; 7,5$; $4,5 \mathrm{~Hz}, \mathrm{H}-3_{\mathrm{A}}$ ), 2,13 (dddd, $J=13,0 ; 8,5 ; 4,5 ; 4,5 \mathrm{~Hz}, \mathrm{H}-3_{\mathrm{B}}$ ), 2,92 ( $m, \mathrm{H}-4), 6,62$ ( $s, \mathrm{H}-5), 7,78$ ( $s, \mathrm{H}-8), 2,18(s, \mathrm{H}-9), 1,29$ ( $d, 6,5 \mathrm{~Hz}$, $\mathrm{H}-10)$. RMN de ${ }^{13} \mathrm{C}\left(125 \mathrm{MHz}, \mathrm{CDCl}_{3}\right) \delta: 197,4$ (C-1), 36,3 (C-2), 30,9 (C-3), 32,6 (C-4), 149,5 (C-4a), 112,8 (C-5), 158,7 (C-6), 125,5 (C-7), 130,6 (C-8), 122,5 (C-8a), 15,1 (C-9), 20,6 (C-10).nb

\section{RESULTADOS E DISCUSSÃO}

O espectro de IV do sesquiterpeno 1 apresentou bandas em 3442, 1633 e $1460 \mathrm{~cm}^{-1}$, consistentes com a presença de hidroxila e grupo fenólico. O espectro de massas HRESI-MS (+), apresentou o pico base $\mathrm{m} / \mathrm{z}, 217,1602(100 \%)$, indicando a formação do aduto $\left[\mathrm{M}-\mathrm{H}_{2} \mathrm{O}+\mathrm{H}\right]^{+}$. A massa para $\left[\mathrm{M}-\mathrm{H}_{2} \mathrm{O}+\mathrm{H}\right]^{+}$foi calculada em 217,1593 o que, com auxílio dos espectros de RMN de ${ }^{13} \mathrm{C}$ e DEPT ( $135^{\circ}$ e $\left.90^{\circ}\right)$, permitiu propor a fórmula molecular $\mathrm{C}_{15} \mathrm{H}_{22} \mathrm{O}_{2}$.

$\mathrm{O}$ espectro de RMN de ${ }^{13} \mathrm{C}$, com auxílio do DEPT $\left(135^{\circ}\right.$ e $\left.90^{\circ}\right)$ indicou a presença de seis carbonos aromáticos $\left(\delta_{\mathrm{C}} 115,1 ; 121,6\right.$; $130,2 ; 130,4 ; 138,6$ e 152,0$)$, quatro metílicos $\left(\delta_{\mathrm{C}} 17,2 ; 21,1 ; 21,4 \mathrm{e}\right.$ 
$15,5)$, quatro metínicos, sendo um carbinólico $\left(\delta_{\mathrm{C}} 41,5 ; 39,0 ; 31,3\right.$ e $71,2)$ e um metilênico $(\delta 26,9)$.

$\mathrm{O}$ espectro de $\mathrm{RMN}$ de ${ }^{1} \mathrm{H}$ de $\mathbf{1}$ revelou a presença de dois singletos, em $\delta_{\mathrm{H}} 6,48(s, 1 \mathrm{H})$ e $\delta_{\mathrm{H}} 6,91(s, 1 \mathrm{H})$, orientados para, evidenciando um sistema aromático tetrassubstituído, também sugerido por RMN de ${ }^{13} \mathrm{C}$. Também apresentou uma porção alifática, com três dubletos, em $\delta_{\mathrm{H}} 1,18\left(d, \mathrm{~J}=7,0 \mathrm{~Hz}, 3 \mathrm{H}, \delta_{\mathrm{C}} 21,4\right), \delta_{\mathrm{H}} 0,67(d, J=6,5$ $\left.\mathrm{Hz}, 3 \mathrm{H}, \delta_{\mathrm{C}} 17,2\right)$, e $\delta_{\mathrm{H}} 0,95\left(\mathrm{~d}, J=7,0 \mathrm{~Hz}, 3 \mathrm{H}, \delta_{\mathrm{C}} 21,1\right)$, além de seis sinais em $\delta_{\mathrm{H}} 2,65\left(q ; J=6,5 \mathrm{~Hz} ; 1 \mathrm{H}, \delta_{\mathrm{C}} 41,5\right), \delta_{\mathrm{H}} 3,88(q ; J=6,5$ $\left.\mathrm{Hz} ; 1 \mathrm{H}, \delta_{\mathrm{C}} 71,2\right), \delta_{\mathrm{H}} 1,72\left(d d d, J=13,0 ; 7,0\right.$ e $\left.6,5 \mathrm{~Hz}, 1 \mathrm{H}, \delta_{\mathrm{C}} 26,9\right)$, $\delta_{\mathrm{H}} 1,75\left(d d d, J=13,0 ; 7,0\right.$ e $\left.3,0 \mathrm{~Hz}, 1 \mathrm{H}, \delta_{\mathrm{C}} 26,9\right), \delta_{\mathrm{H}} 2,72\left(m, 1 \mathrm{H}, \delta_{\mathrm{C}}\right.$ $39,0)$ e $\delta_{\mathrm{H}} 2,20\left(m, 1 \mathrm{H}, \delta_{\mathrm{C}} 31,3\right)$. As correlações ${ }^{1} \mathrm{H}-{ }^{1} \mathrm{H}$ observadas no experimento de $g \mathrm{COSY}$, entre $\mathrm{H}-14 / \mathrm{H}-10, \mathrm{H}-10 / \mathrm{H}-9, \mathrm{H}-9 / \mathrm{H}-8$, H-8/H-7, H-7/H-11, H-11/H-12 e H-11/H-13, aliadas às correlações observadas nos mapas de contorno dos experimentos de $g \mathrm{HMBC}$ entre $\mathrm{H}-14 \leftrightarrow \mathrm{C}-10 / \mathrm{C}-9, \mathrm{H}-12$ e $13 \leftrightarrow \mathrm{C}-11 / \mathrm{C}-7$ permitiram propor a unidade alifática para o sesquiterpeno $\mathbf{1}$. Com base nas análises dos mapas de contorno do experimento $g \mathrm{HMQC}$, foi possível atribuir todos os hidrogênios aos respectivos carbonos.

As correlações ${ }^{3} J$ e ${ }^{2} J$ observadas no experimento $g \mathrm{HMBC}$ de $\mathrm{H}-2 \leftrightarrow \mathrm{C}-3 / \mathrm{C}-1 / \mathrm{C}-6$ e de $\mathrm{H}-5 \leftrightarrow \mathrm{C}-1 / \mathrm{C}-3 / \mathrm{C}-15$, permitiram posicionar os substituintes no anel aromático, sendo corroborado pela correlação espacial observada em NOESY 1D, entre H-5 e Me-15. Os valores de deslocamento químico observados em $\mathrm{RMN}$ de ${ }^{13} \mathrm{C}$ para este padrão de substituição aromático estão de acordo com modelos da literatura. ${ }^{14}$

A junção dos anéis entre C-10 e C-1 foi realizada mediante as correlações observadas no experimento de HMBC entre $\mathrm{H}-14 \leftrightarrow \mathrm{C}-1 \mathrm{e}$ a correlação espacial de $\mathrm{H}-2\left(\delta_{\mathrm{H}} 6,48\right) \operatorname{com} \delta_{\mathrm{H}} 1,18(\mathrm{Me}-14)$ e com $\delta_{\mathrm{H}}$ 2,65 (H-10), no experimento NOESY. A ciclização do anel alifático foi efetuada pela correlação entre $\mathrm{H}-5 \leftrightarrow \mathrm{C}-7$ por HMBC e, também, através da correlação espacial entre H-5 e H-7, observada por NOESY.

A estereoquímica relativa de $\mathbf{1}$ foi estabelecida com base nas interações observadas em NOESY entre H-14 $\left(\delta_{\mathrm{H}} 1,18\right)$ com H-13 $\left(\delta_{\mathrm{H}} 0,95\right)$ e $\mathrm{H}-12\left(\delta_{\mathrm{H}} 0,65\right)$, colocando $\mathrm{H}-14$ e o grupo isopropil em uma relação cis.

A configuração relativa de C-9 foi realizada por HOMODEC, onde irradiação em H-10 mostrou H-9 como um duplo dubleto largo em $\delta_{\mathrm{H}} 3,88\left(J_{9 e q, 8 a x} 3,0 \mathrm{~Hz}\right.$ e $\left.J_{9 e q, 8 e q} 2,5 \mathrm{~Hz}\right)$. Estas observações foram confirmadas por NOESY utilizando $\mathrm{C}_{5} \mathrm{D}_{5} \mathrm{~N}$, sendo observada a interação entre H-12 e H-11 com H-9, colocando-os em uma relação 1,3 pseudo diaxial ( cis) e permitindo estabelecer a estereoquímica relativa de 1. Esta é uma nova substância denominada Rel.(-)-(7R,9S,10R)3,9-di-hidroxicalameneno, estereoisômero de Rel. (7R,9S,10S)3,9-di-hidroxicalameneno isolado de Heterotheca subaxiliaris. ${ }^{15}$
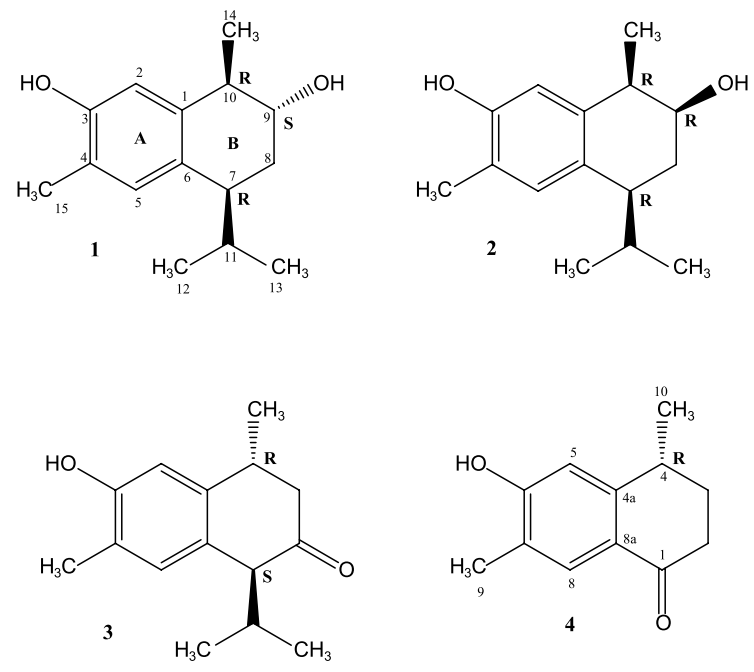

Figura 1. Substâncias produzidas por Phomopsis cassiae
O espectro de massas de 3,9-di-hidroxicalameneno (2) HRESIMS (+) apresentou o pico base $m / z, 217,1593$ (100\%), indicando a formação do aduto $\left[\mathrm{M}-\mathrm{H}_{2} \mathrm{O}+\mathrm{H}\right]^{+}$. A massa para $\left[\mathrm{M}-\mathrm{H}_{2} \mathrm{O}+\mathrm{H}\right]^{+}$foi calculada em 217,15924, o que com auxílio dos espectros de RMN de ${ }^{13} \mathrm{C}$ e DEPT $\left(90^{\circ}, 135^{\circ}\right)$ permitiu confirmar a fórmula molecular $\mathrm{C}_{15} \mathrm{H}_{22} \mathrm{O}_{2}$.

As análises dos dados espectroscópicos de RMN de ${ }^{1} \mathrm{H},{ }^{13} \mathrm{C}$, $g \mathrm{HMQC}, g \mathrm{HMBC}$ e $g \mathrm{COSY}$ indicaram que $\mathbf{2}$ possui a mesma estrutura planar que $\mathbf{1}$. A estereoquímica relativa de $\mathbf{2}$ foi determinada através do experimento NOESY em $\mathrm{C}_{5} \mathrm{D}_{5} \mathrm{~N}$, sendo observadas interações H-14 e H-12, permitindo posicioná-los, assim como 1, em uma relação cis. A estereoquímica relativa de $\mathrm{H}-9$ foi definida com base nas constantes de acoplamento entre $\mathrm{H}-9$ e $\mathrm{H}-10 / \mathrm{H}-8$, onde se verificou um duplo tripleto em $\delta 3,99\left(J_{9 a x, 8 a x} 12,0 \mathrm{~Hz} ; J_{9 a x, 8 e q} 4,5 \mathrm{~Hz} ; J_{9 a x, 10 \mathrm{eq}} 4,5\right.$ $\mathrm{Hz}$ ), estabelecendo a configuração entre $\mathrm{H}-9$ e $\mathrm{H}_{3} \mathrm{C}-14$. A substância

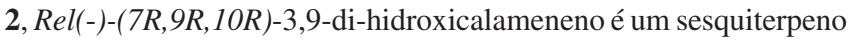
inédito na literatura, também estereoisômero de Rel. (7R,9S, 10S)-dihidroxicalameneno, isolado de Heterotheca subaxiliaris. ${ }^{15}$

A estrutura da substância 3 foi definida com base na análise dos espectros de $\mathrm{RMN}$ de ${ }^{1} \mathrm{H}$ e de ${ }^{13} \mathrm{C}$ uni e bidimensionais e comparação com valores descritos na literatura. ${ }^{16} \mathrm{~A}$ substância 3 foi isolada como um óleo amarelo claro $(2,3 \mathrm{mg}) ;[\alpha]^{25}{ }_{\mathrm{D}}+9,9(c 0,25, \mathrm{MeOH})$. Submetida à espectrometria de massas HRESI-MS (-) apresentou o pico base $m / z 233,1542(100 \%)$ relativo à formação do aduto $[\mathrm{M}+\mathrm{H}]^{+}$.

As análises dos dados de RMN de ${ }^{1} \mathrm{H}, \mathrm{HMQC}, \mathrm{HMBC}$ e COSY de $\mathbf{3}$ evidenciaram uma grande semelhança com $\mathbf{1}$ e $\mathbf{2}$, sugerindo tratarem-se de análogos. A ausência de H-8 carbinólico em $\delta_{\mathrm{H}} 3,99$ $\left(\delta_{\mathrm{C}} 71,4\right)$ e a presença de uma carbonila em $\delta_{\mathrm{C}} 213,0$ evidenciaram uma diferenciação estrutural. $\mathrm{O}$ posicionamento da carbonila em C-8 foi definido pela análise dos dados de COSY e HMBC. No primeiro, foram visualizadas as correlações de $\mathrm{H}-10 \leftrightarrow \mathrm{H}-9 / \mathrm{H}-14$, $\mathrm{H}-12 \leftrightarrow \mathrm{H}-11 / \mathrm{H}-13, \mathrm{H}-11 \leftrightarrow \mathrm{H}-7 / \mathrm{H}-12 / \mathrm{H}-13$, que aliadas às observadas em $g \mathrm{HMBC}$ de $\mathrm{H}-10 \leftrightarrow \mathrm{C}-9 / \mathrm{C}-8, \mathrm{H}-11 / \mathrm{C}-8$ permitiram o posicionamento inequívoco da carbonila em C-8. Esta constatação foi corroborada pela observação dos duplos dubletos em $\delta_{\mathrm{H}} 2,62(d d ; J$ $=17,5 ; 5,5 \mathrm{~Hz} ; 1 \mathrm{H})$ e $\delta_{\mathrm{H}} 2,05(d d ; J=17,5 ; 11,5 \mathrm{~Hz} ; 1 \mathrm{H})$, atribuídos a $\mathrm{H}-9_{\mathrm{ax}}$ e $\mathrm{H}-9_{\mathrm{eq}}$, em posição vicinal a $\mathrm{H}-10$.

A configuração relativa de $\mathbf{3}$ foi definida após avaliação dos experimentos de NOESY, onde se observou uma forte correlação entre $\mathrm{H}-10$ e $\mathrm{H}_{3} \mathrm{C}-11$, permitindo posicionar o grupo isopropil em relação trans a $\mathrm{H}_{3} \mathrm{C}-14$. Esta substância foi identificada como Rel. $(+)(7 S, 10 R)$-3-hidroxicalamen-8-ona (3), previamente isolada do coral australiano Lemnalia cervicornis. ${ }^{16}$

A estrutura da substância $\mathbf{4}$ foi definida com base na análise dos espectros de RMN de ${ }^{1} \mathrm{H}$ e de ${ }^{13} \mathrm{C}$ uni e bidimensionais e comparação com valores descritos na literatura. ${ }^{17}$ Esta foi isolada como um óleo amarelo claro $(2,3 \mathrm{mg}) \mathrm{e}[\alpha]^{25}+32,6(c 0,25, \mathrm{MeOH})$. Submetida à espectrometria de massas HRESI-MS (+) apresentou o pico base $m / z$. $191,10728(100 \%)$ relativo à formação do aduto $[\mathrm{M}+\mathrm{H}]^{+}$.

$\mathrm{O}$ espectro de $\mathrm{RMN}$ de ${ }^{1} \mathrm{H}$ de $\mathbf{4}$ apresentou dois singletos em $\delta_{\mathrm{H}}$ $6,62(s ; 1 \mathrm{H})$ e $\delta_{\mathrm{H}} 7,78(s, 1 \mathrm{H})$, sugerindo um anel aromático tetrassubstituído, com dois hidrogênios orientados para e um singleto em $2,18(s, 3 \mathrm{H})$, atribuído a uma metila aromática. Foram visualizados também dois duplos duplos dubletos em $\delta_{\mathrm{H}} 2,47$ ( $d d d ; J=17,0 ; 8,5$ e 4,5 Hz; 1H) e $\delta_{\mathrm{H}} 2,65$ ( $d d d ; J=17,0 ; 8,0$ e 4,5 Hz; $\left.1 \mathrm{H}\right)$, atribuídos aos hidrogênios geminais $\mathrm{H}-2$. Na região alifática, foram observados um dupleto em $\delta_{\mathrm{H}} 1,29(d ; J=6,5 \mathrm{~Hz} ; 3 \mathrm{H})$, um multipleto em $\delta_{\mathrm{H}}$ $2,92(m, 1 \mathrm{H})$ e dois sinais em $\delta_{\mathrm{H}} 2,13(d d d d ; J=13,0 ; 8,5 ; 7,5$ e 4,5 $\mathrm{Hz}, 1 \mathrm{H})$ e $\delta_{\mathrm{H}} 1,79(d d d d ; J=13,0 ; 8,5 ; 4,5$ e $4,5 \mathrm{~Hz} 1 \mathrm{H})$, atribuídos a $\mathrm{H}_{3} \mathrm{C}-10, \mathrm{H}-4$ e aos hidrogênios diastereotópicos $\mathrm{H}-3$.

$\mathrm{O}$ espectro de $\mathrm{RMN}$ de ${ }^{13} \mathrm{C}$ indicou a presença de seis carbonos aromáticos, um metílico aromático e outro alifático, um metínico benzílico, dois metilênicos e um carbonílico, confirmando a 
aromaticidade de 4. Com base na análise do mapa de contorno do experimento $g \mathrm{HMQC}$ foi possível atribuir os átomos de hidrogênios aos respectivos carbonos.

As correlações ${ }^{1} \mathrm{H}-{ }^{1} \mathrm{H}$ observadas no experimento COSY entre H-10/H-4 e H-2/H-3, juntamente com as correlações observadas no experimento $g \mathrm{HMBC}$ entre $\mathrm{H}-2 \leftrightarrow \mathrm{C}-1 / \mathrm{C}-3 / \mathrm{C}-4, \mathrm{H}-4 \leftrightarrow \mathrm{C}-4 \mathrm{a}$,

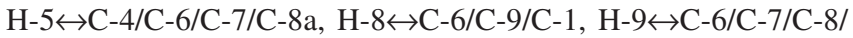
$\mathrm{C}-8^{\mathrm{a}}$ e $\mathrm{H}-10 \leftrightarrow \mathrm{C}-3 / \mathrm{C}-4$, permitiram a confirmação dos valores atribuídos aos carbonos e estabelecer a estrutura planar, identificando a substância $\mathbf{4}$ como a tetralona aristelegona-A, isolada de Aristolochia elegans. ${ }^{17}$

As substâncias 1-4 foram submetidas a ensaio para avaliação das atividades antifúngica contra os fungos fitopatogênicos Cladosporium cladosporioides e C. sphaerospermum e anticolinesterásica. Os resultados dos limites de detecção das substâncias 1-4 estão apresentados na Tabela 1. Este é o primeiro relato do potencial antifúngico e anticolinesterásico demonstrado por estas substâncias.

Tabela 1. Valores dos limites de detecção $(\mu \mathrm{g})$ obtidos para os ensaios contra os fungos fitopatogênicos C. cladosporioides e C. sphaerospermum e da avaliação da atividade anticolinesterásica

\begin{tabular}{lccc}
\hline Substâncias & C. cladosporioides & C. sphaerospermum & $\begin{array}{c}\text { Enzima } \\
\text { AChE }\end{array}$ \\
\hline $\mathbf{1}$ & 5 & 5 & 3 \\
$\mathbf{2}$ & 5 & 5 & 3 \\
$\mathbf{3}$ & 5 & 5 & 3 \\
$\mathbf{4}$ & 5 & 5 & 3 \\
galantamina & - & - & 1 \\
nistatina & 1 & 1 & - \\
\hline
\end{tabular}

\section{CONCLUSÃO}

As substâncias 3-hidroxicalamen-8-ona (3) e aristelegona (4) são descritas do coral australiano Lemnalia cervicornis ${ }^{16}$ e das raízes e talos de Aristolochia elegans, ${ }^{17}$ sendo este o primeiro relato da produção destes metabólitos por fungos.

As atividades antifúngica e anticolinesterásica apresentadas pelas substâncias 1-4 reforçam as ressalvas que fungos endofíticos são prolíficos produtores de metabólitos bioativos, além de sugerirem uma possível relação de simbiose entre a espécie hospedeira Cassia spectabilis e o fungo endofítico Phomopsis cassiae, este produzindo substâncias bioativas contra possíveis fitopatógenos.

$P$. cassiae é um fungo endofítico que tem sido objeto de estudo pelo nosso grupo de pesquisa apresentando resultados excelentes: quando cultivado em meio líquido MDB, produziu sesquiterpenos da classe dos cadalenos, calamenenos ${ }^{10}$ e policetídeos, ${ }^{7}$ todos inéditos, com atividade contra os fungos fitopatogênicos Cladosporium cladosporioides e $C$. sphaerospermum, e citotoxicidade contra a linhagem celular de tumor cervical humano (HeLa). Este é o primeiro relato do cultivo deste endófito em extrato de malte e os resultados alcançados reforçam as observações que a produção metabólica dos micro-organismos é dependente do meio de cultivo. Com este trabalho queremos evidenciar a urgente necessidade no estudo deste nicho de micro-organismos, considerando a crescente taxa de extinção de espécies vegetais brasileiras.

\section{MATERIAL SUPLEMENTAR}

Está disponível em http://quimicanova.sbq.org.br, em arquivo PDF, com acesso livre.

\section{AGRADECIMENTOS}

À FAPESP, programa Biota-FAPESP (Instituto Virtual da Biodiversidade, www.biota.org) pelo auxílio financeiro, à CAPES pela bolsa concedida a H. L. Teles e ao CNPq pelas bolsas concedidas a L. M. Zanardi, G. H. Silva, V. da S. Bolzani e M. C. M. Young.

\section{REFERÊNCIAS}

1. Kharwar, R. N.; Mishra, A.; Gond, S. K.; Stierle, A.; Stierle, D.; Nat. Prod. Rep. 2011, 28, 1208.

2. Souvik, K. S.; Spiteller, M.; Nat. Prod. Rep. 2011, 28, 1203.

3. Aly, A. M.; Debbab, A.; Proksch, P.; Appl Microbiol Biotechnol. 2011, 90, 1829.

4. Gunatilaka, A. A. L.; J. Nat. Prod. 2006, 69, 509.

5. Strobel, G. A.; Daisy, B.; Castillo, U.; Harper, J.; J. Nat. Prod. 2004, 67, 257.

6. Tan, R. X.; Zou, W. X.; Nat. Prod. Rep. 2001, 18, 448.

7. Silva, G. H.; Teles, H. L.; Trevisan, H. C.; Young, M. C. M.; Pfenning, L. H.; Eberlin, M. N.; Haddad, R.; Costa Neto, C.; Bolzani, V. S.; Araújo, A. R.; J. Braz. Chem. Soc. 2005, 16, 1463.

8. Teles, H. L.; Silva, G. H.; Castro-Gamboa, I.; Bolzani, V. S.; Pereira, J. O.; Costa Neto, C.; Haddad, R.; Eberlin, M. N.; Young, M. C. M.; Araújo, A. R.; Phytochemistry 2005, 66, 2363.

9. Cafêu, M. C.; Silva, G. H.; Teles, H. L.; Bolzani, V. S.; Araújo, A. R.; Young, M. C.M.; Pfenning, L. H.; Quim. Nova 2005, 28, 991.

10. Silva, G. H.; Teles, H. L.; Zanardi, L. M.; Young, M. C.M.; Haddad, R.; Eberlin, M. N.; Pfenning, L. H.; Claudio Costa-Neto, C. M.; CastroGamboa, I.; Bolzani, V. da S.; Araújo, A. R.; Phytochemistry 2006, 67, 1964.

11. Inácio, M. L.; Silva, G. H.; Teles, H. L.; Trevisan, H. C.; Cavalheiro, A. J.; Bolzani, V. da S.; Young, M. C. M.; Pfenning, L. H.; Araújo, A. R.; Biochem. System. Ecol. 2006, 34, 822.

12. Rhalison, L.; Hamburger, M.; Hostettmann, K.; Monod, M.; Frenk, E.; Phytochem. Anal. 1991, 5, 199.

13. Marston, A.; Kissling, J.; Hostettmann, K.; Phytochem. Anal. 2002, 13, 51 .

14. Nabeta, K.; Katayama, K.; Nakagawara, S.; Katoh, K.; Phytochemistry 1993, 32, 117.

15. Bohlmann, F.; Zdero, C.; Phytochemistry 1979, 18, 1185

16. Bowden, B. F.; Coll, J. C.; Engelhardt, L. M.; Tapiolas, D. M.; White, A. H.; Aust. J. Chem. 1986, 39, 103.

17. Wu, T.S.; Tsai, Y. L.; Damu, A. G.; Kuo, P. C.; Wu, P. L.; J. Nat. Prod. 2002, 65, 1522 . 
SESQUITERPENOS PRODUZIDOS PELO FUNGO ENDOFÍTICO Phomopsis cassiae COM ATIVIDADE ANTIFÚNGICA E INIBIDORA DE ACETILCOLINESTERASE ${ }^{\sharp}$

Lisinéia M. Zanardi, Vanderlan da S. Bolzani, Alberto J. Cavalheiro, Dulce H. Siqueira Silva, Henrique C. Trevisan ${ }^{\dagger}$ e Angela R. Araujo*

Instituto de Química, Universidade Estadual Paulista, CP 355, 14801-970 Araraquara - SP, Brasil

Geraldo H. Silva

Centro de Ciências Exatas e Tecnologia, Universidade Federal de Sergipe, 49100-000 Aracajú - SE, Brasil

Helder L. Teles

Departamento de Ciências Biológicas, Universidade Federal do Mato Grosso, 78735-901 Rondonópolis - MT, Brasil

Maria Cláudia M. Young

Secção de Fisiologia e Bioquímica de Plantas, Instituto de Botânica, CP 4005, 01061-970 São Paulo - SP, Brasil
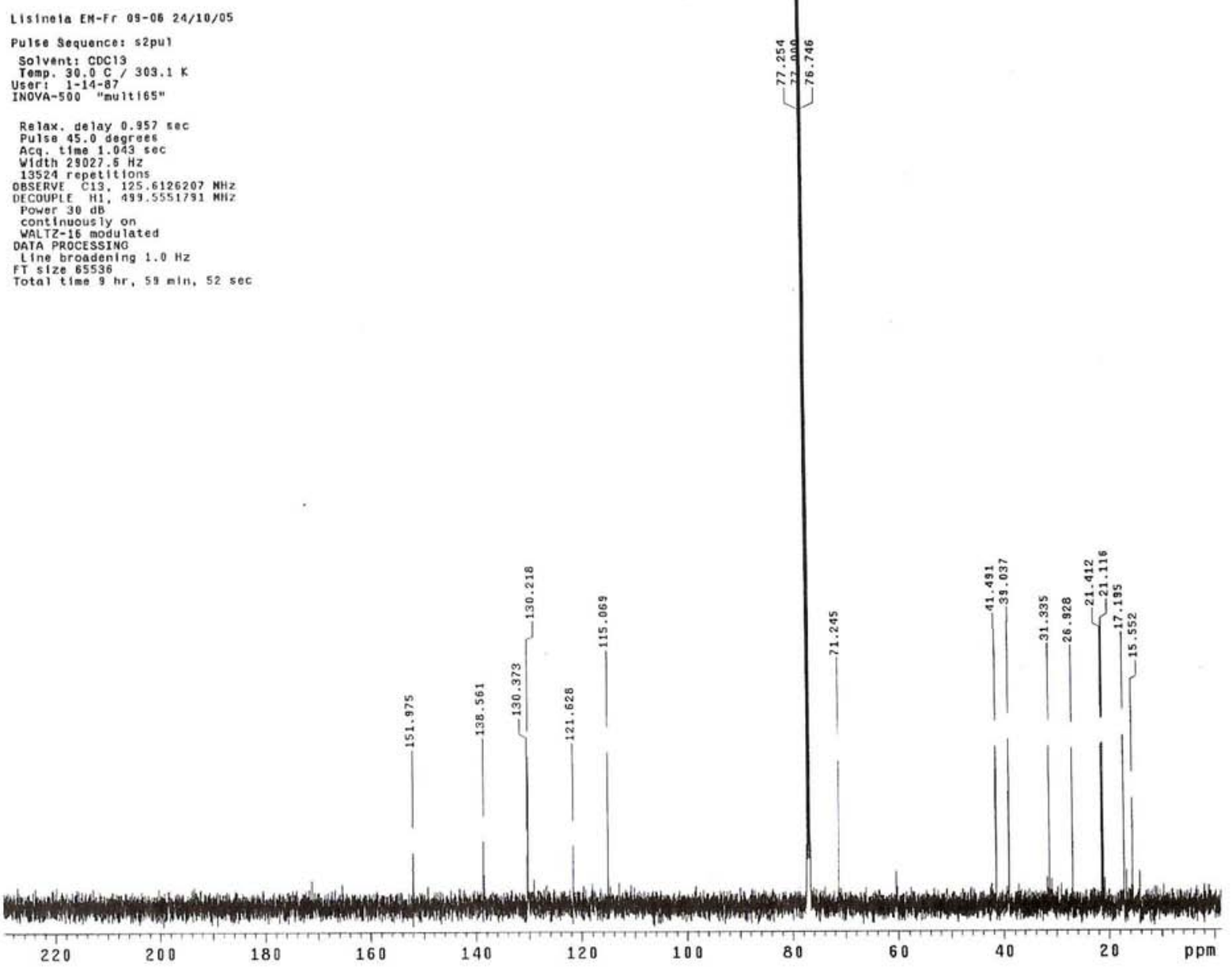

Figura 1S. Espectro de $\mathrm{RMN} \mathrm{de}{ }^{13} \mathrm{C}$ de $1\left(\mathrm{CDCl}_{3}, 125 \mathrm{MHz}\right)$

*e-mail: araujoar@iq.unesp.br

†n Memoriam

\#Artigo em homenagem ao Prof. Otto R. Gottlieb (31/8/1920-19/6/2011) 
Listneia E.M. Fr 09-06 16/09/05

Pulse Sequence: s2pul

Solvent: COC13
Temp. $30.0 \mathrm{C}$. $303.1 \mathrm{~K}$
INOVn-500

Relax, delay $0.904 \mathrm{sec}$

Pulse 45.0 degrees

Width ${ }^{\text {time }} 41.105$ s

16 repotations

199.5594831 naz

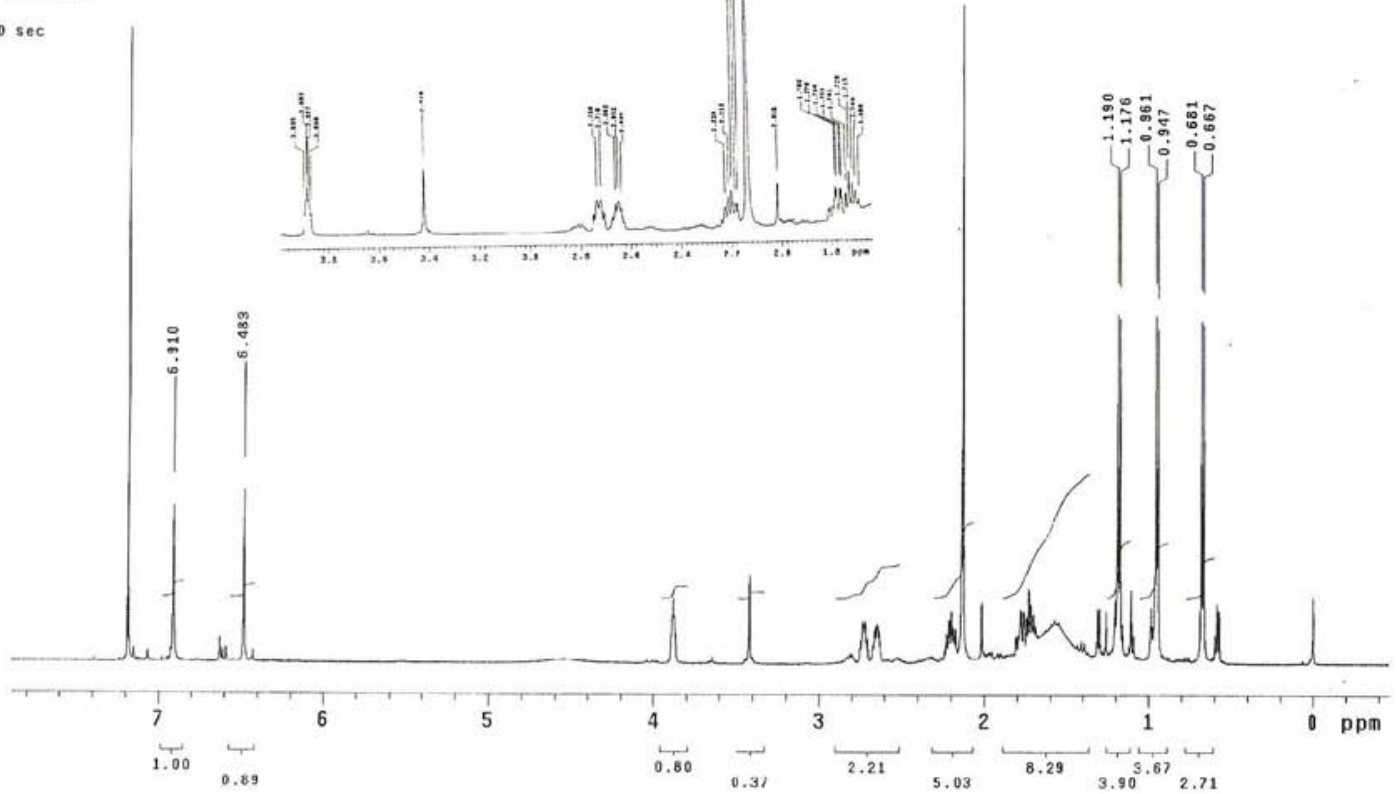

Figura 2S. Espectro de $\mathrm{RMN} \mathrm{de}{ }^{1} \mathrm{H}$ de $\mathbf{1}\left(\mathrm{CDCl}_{3}, 500 \mathrm{MHz}\right)$

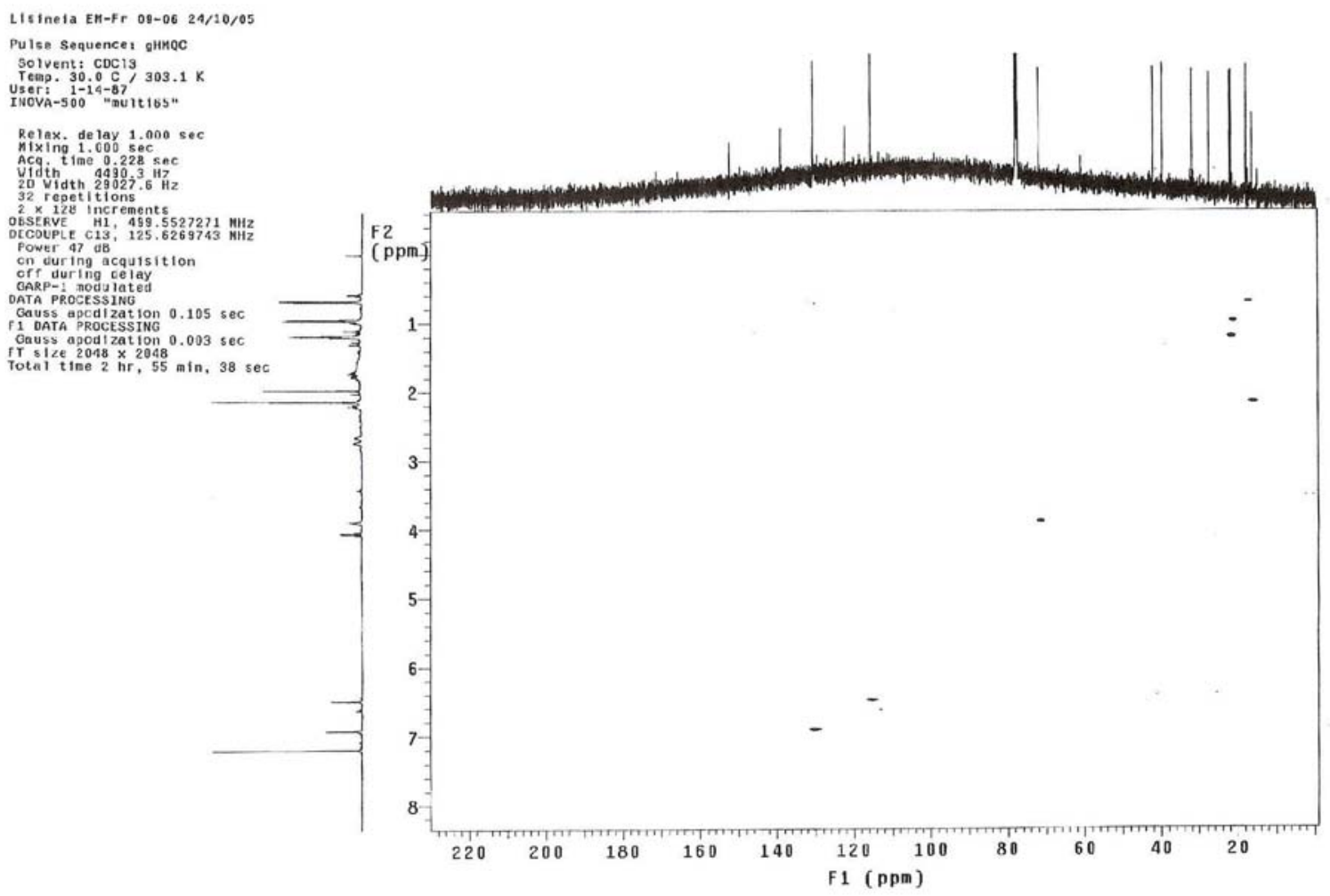

Figura 3S. Mapa de contorno de gHMQC de $1\left(\mathrm{CDCl}_{3}, 125 \mathrm{MHz}\right)$ 


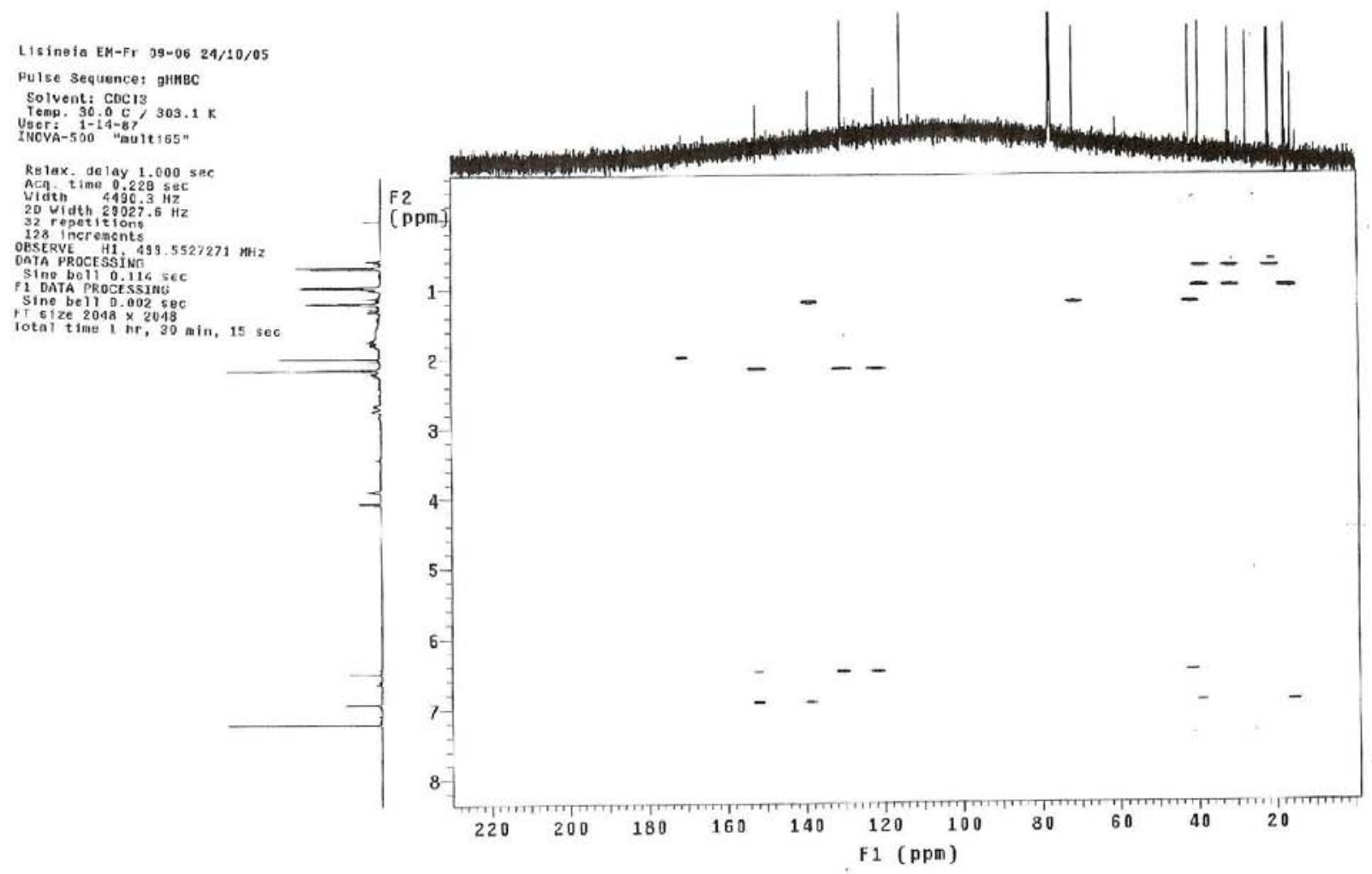

Figura 4S. Mapa de contorno de gHMBC de $1\left(\mathrm{CDCl}_{3}, 125 \mathrm{MHz}\right)$ 
Lisineta en ir 09-06 28/09/05

Pulse Sequence: MOESY1D

a)

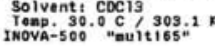

Relax. Jelay $1.000 \mathrm{sec}$

Relax.

Nixing 1.000 ofec

Giditn $4171, \mathrm{O}_{\mathrm{Hz}}$

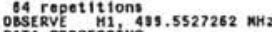

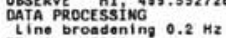

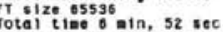

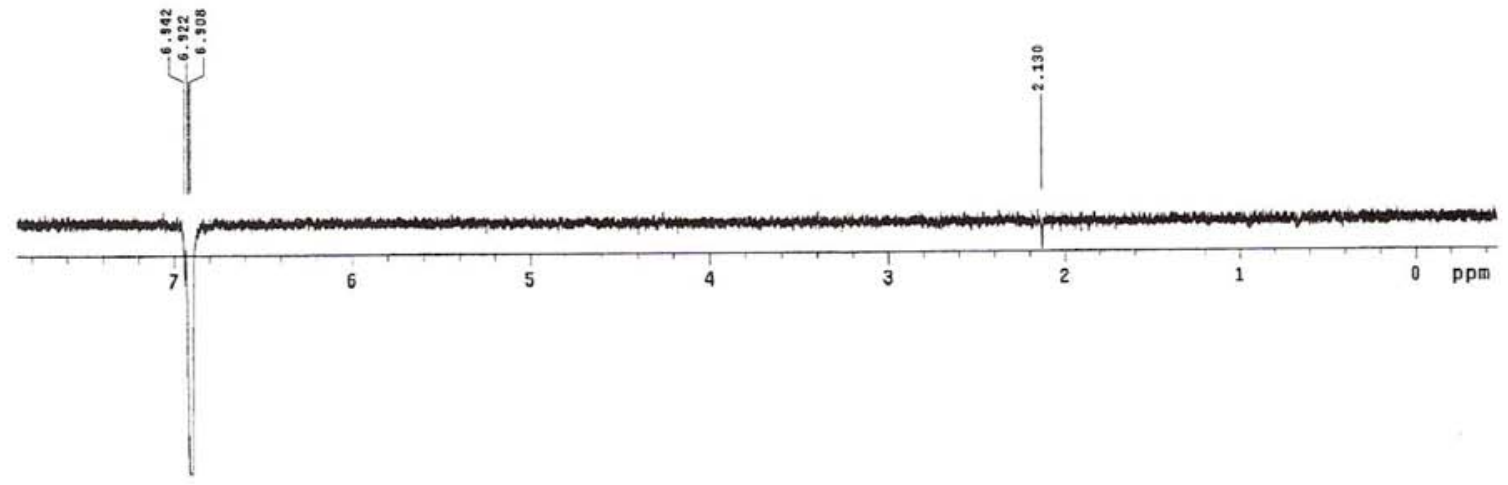

Lisine 10 EM fr 09-06 20/09/0

b)

Pulso Sequence: NOESY10

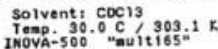

Relax. gelay 1,000 see

Wixing $1: 000$ :

Ace time 4.105 se

su repetitions

OESERVY H11 499.5527262 NHE

OAIA Processing $1.0 \mathrm{~Hz}$

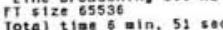

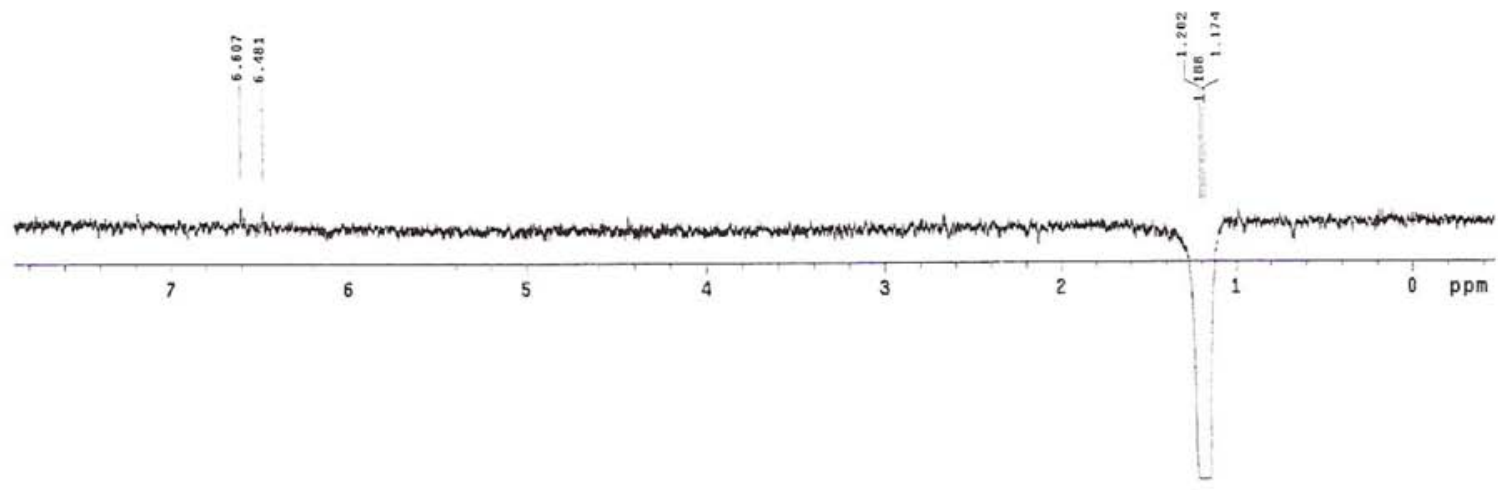

Figura 5S. Espectro de NOESY $1 \mathrm{D}$ de $1\left(\mathrm{CDCl}_{3}, 500 \mathrm{MHz}\right)$, hidrogênios irradiados: a) $\left.\mathrm{H}-6, \delta_{H} 6,91 ; b\right) \mathrm{H}-14, \delta_{H} 1,18$ 
Lisinoia Ext N Fr-00-06(4) 23/02/06

Pulce sequencer notsyido

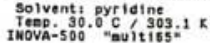

Rolax. golay 1.000 coc

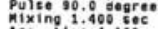

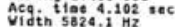

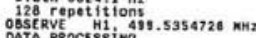

DATA Processino $1.0 \mathrm{Kz}$

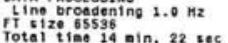

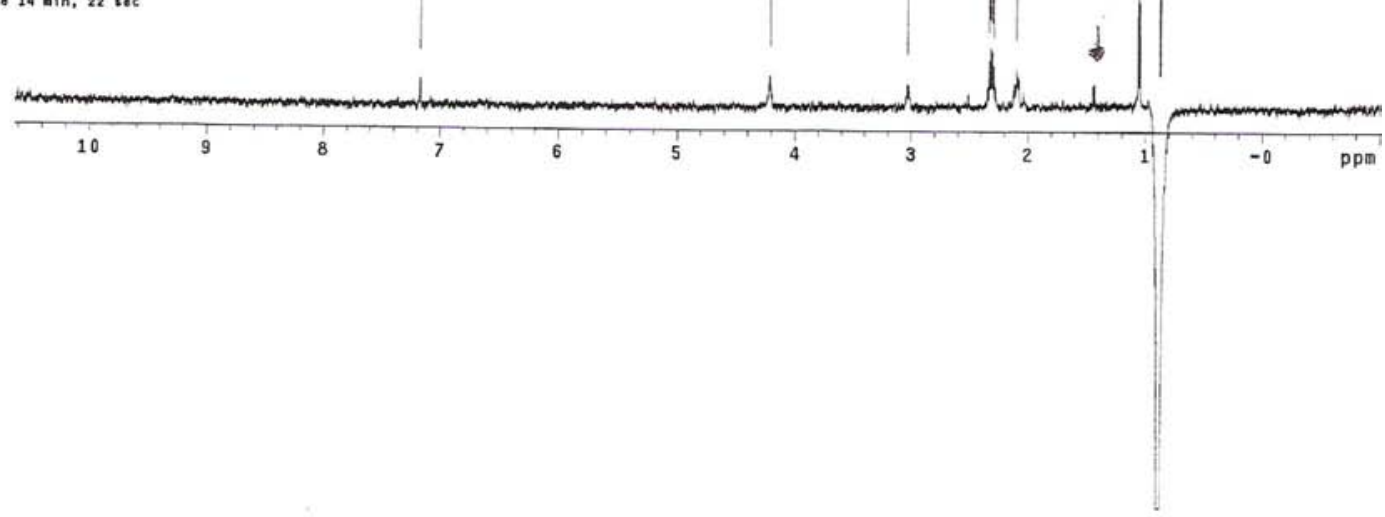

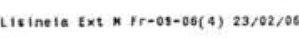

Pulse sequence: notsyid

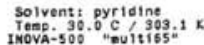

b)

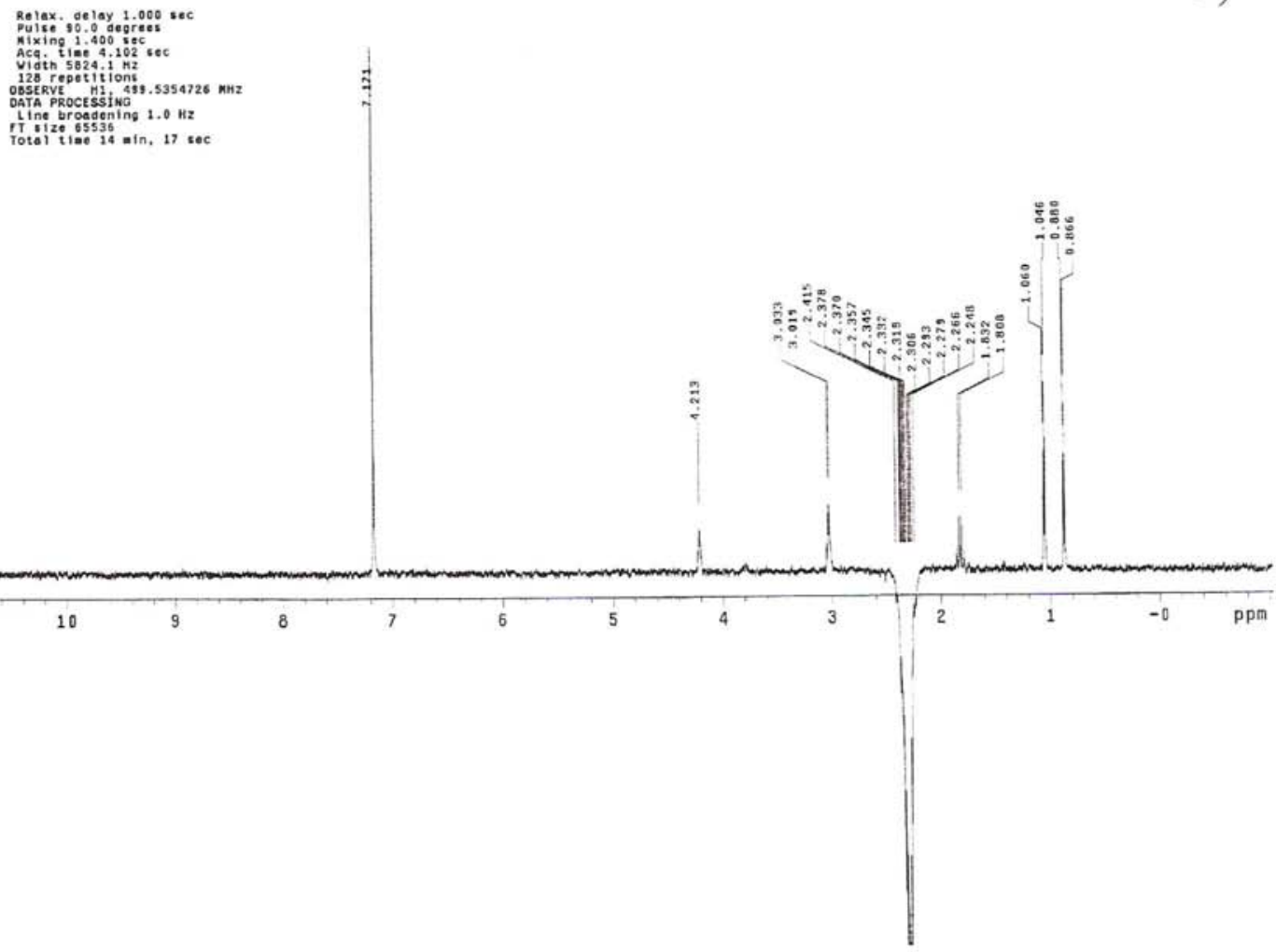

Figura 6S. Espectro de NOESY $1 D$ de $1\left(C_{5} D_{5} N, 500 \mathrm{MHz}\right)$, hidrogênios irradiados: a) $\left.H-12, \delta_{H} 0,87 ; b\right) H-11, \delta_{H} 2,32$ 


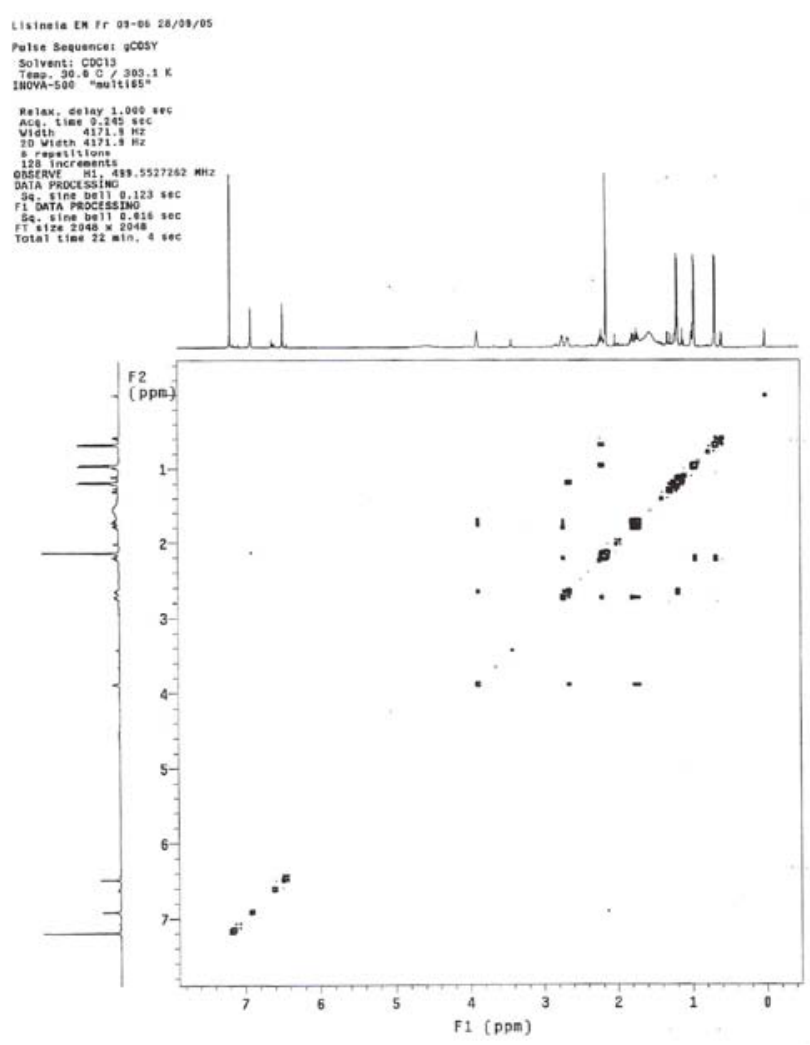

Figura 7S. Espectro de gCOSY de $1\left(\mathrm{CDCl}_{3}, 500 \mathrm{MHz}\right)$

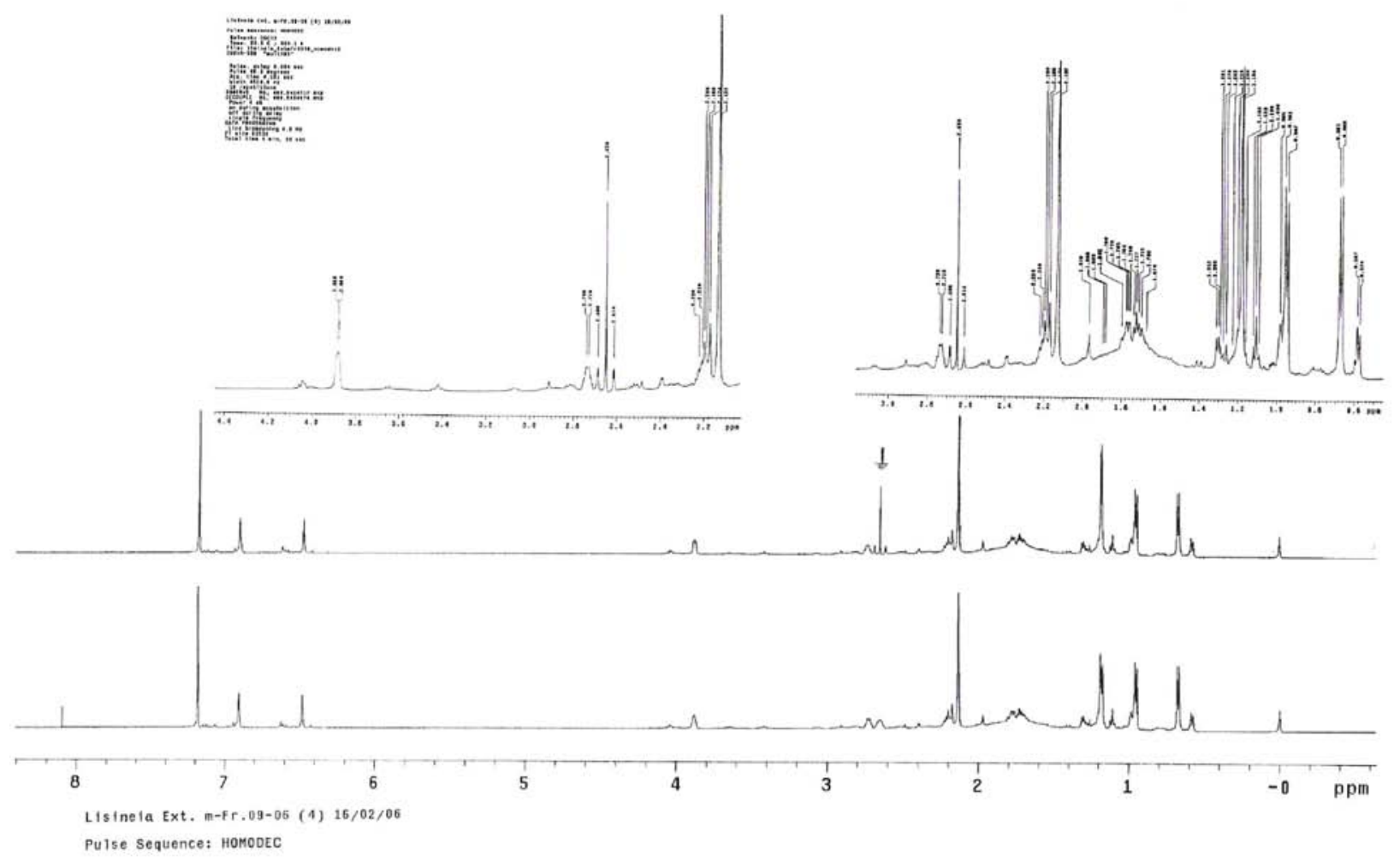

Figura 8S. Espectro de HOMODEC de $\mathbf{1}\left(\mathrm{CDCl}_{3}, 500 \mathrm{MHz}\right)$, hidrogênios irradiados $\mathrm{H}-1, \delta_{H} 2,65$ 


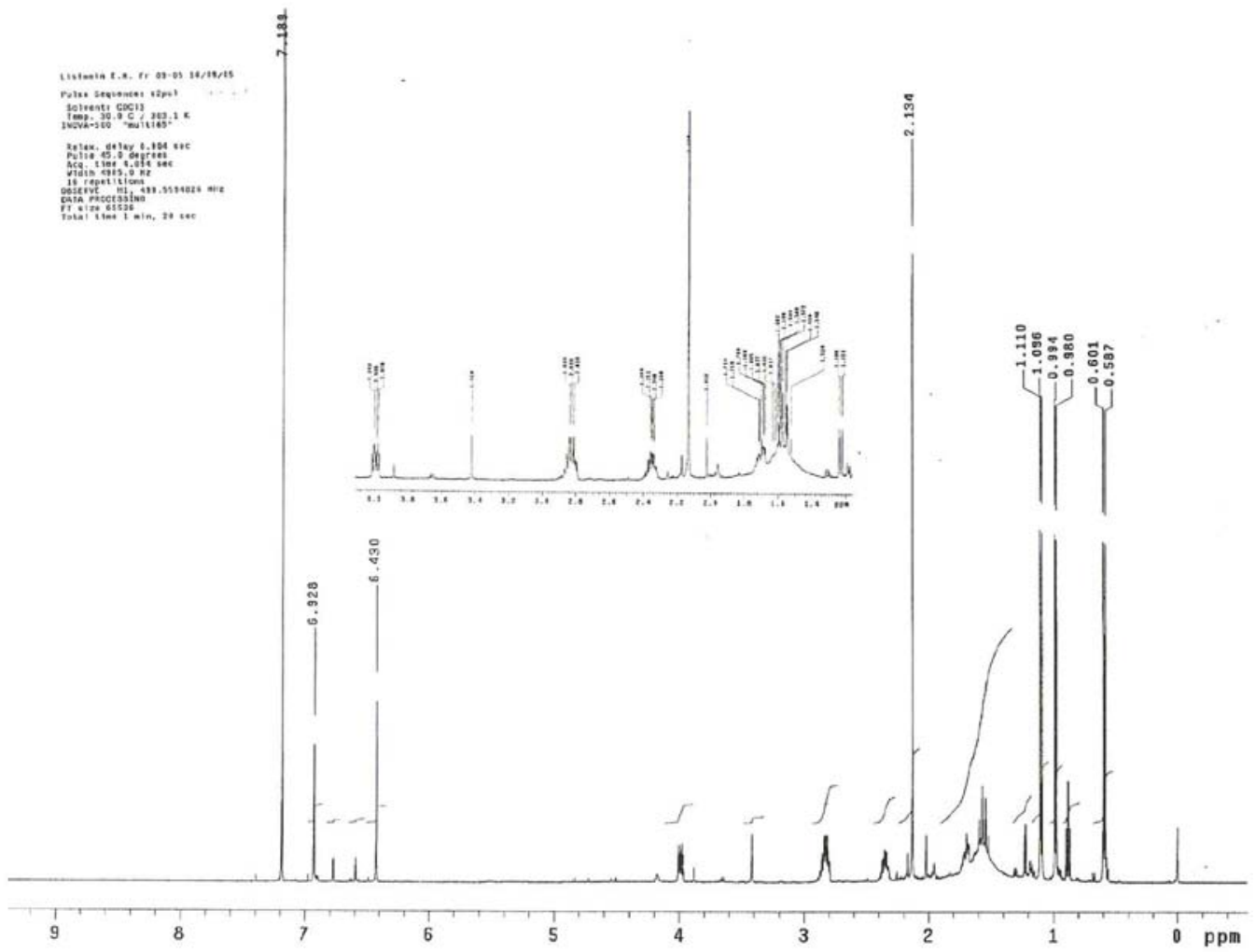

Figura 9S. Espectro de $\mathrm{RMN} \mathrm{de}{ }^{l} \mathrm{H}$ de $2\left(\mathrm{CDCl}_{3}, 500 \mathrm{MHz}\right)$
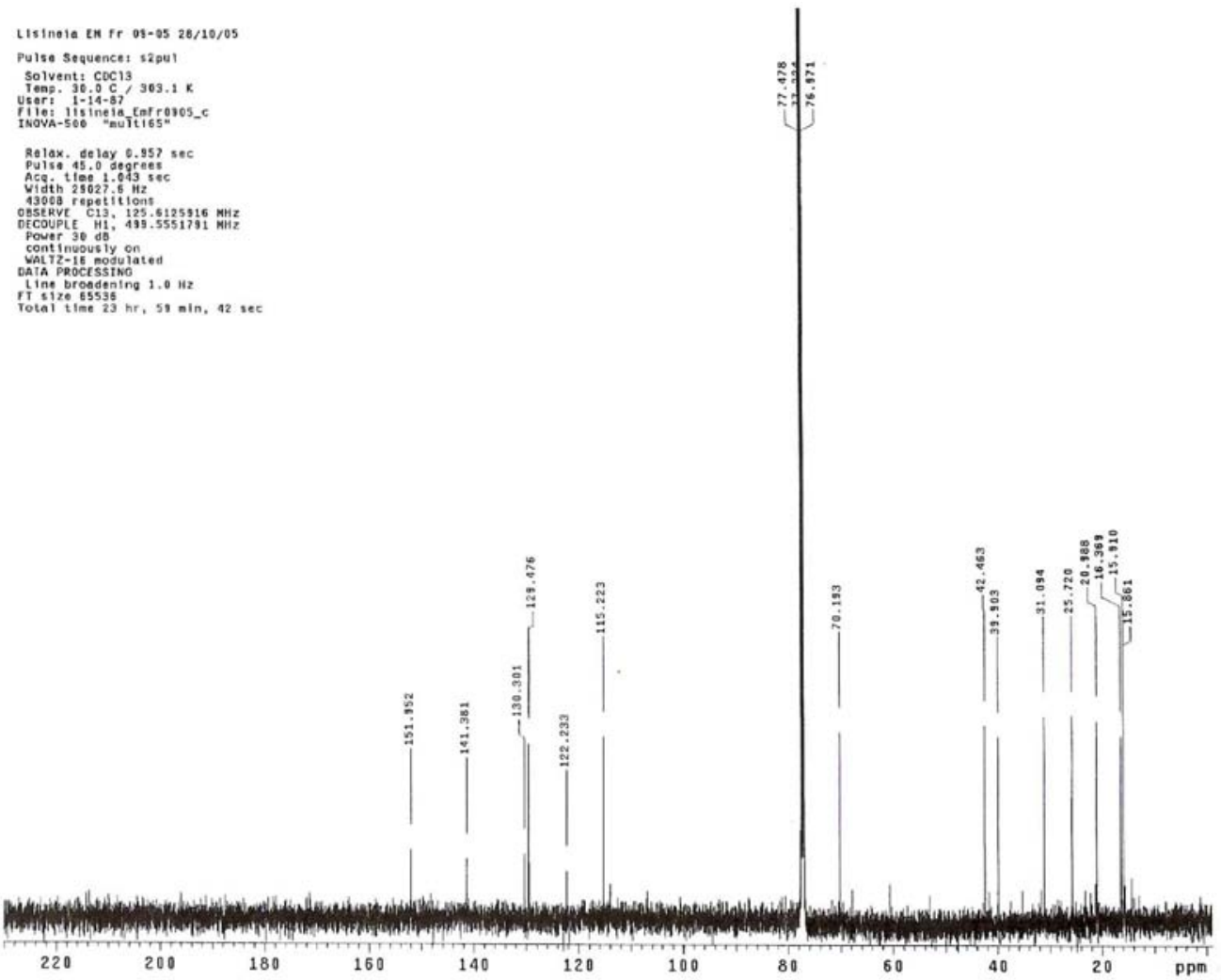

Figura 10S. Espectro de $\mathrm{RMN}$ de ${ }^{13} \mathrm{C}$ de $2\left(\mathrm{CDCl}_{3}, 125 \mathrm{MHz}\right)$ 


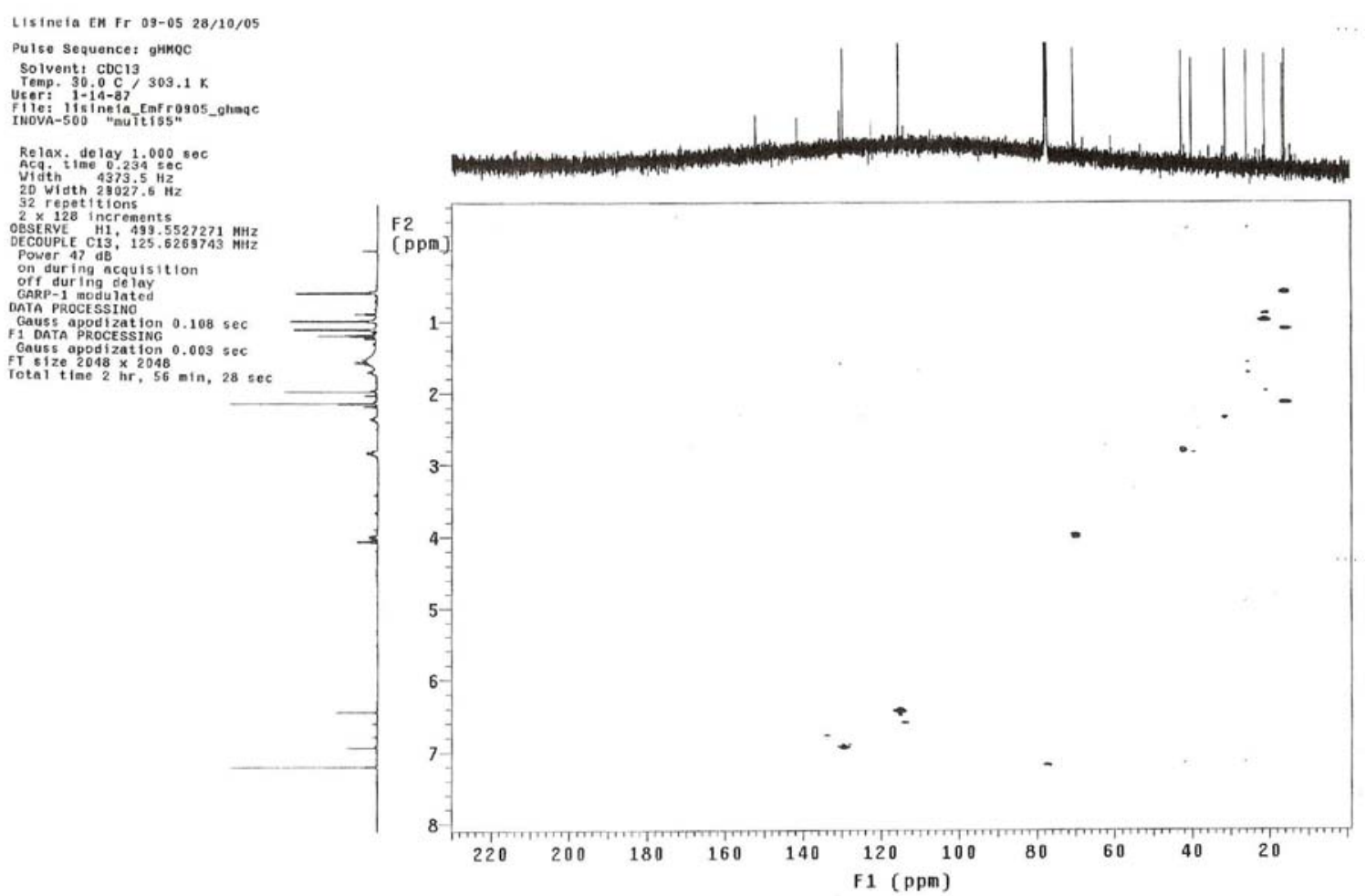

Figura 11S. Mapa de contorno de gHMQC de $2\left(\mathrm{CDCl}_{3}, 125 \mathrm{MHz}\right)$

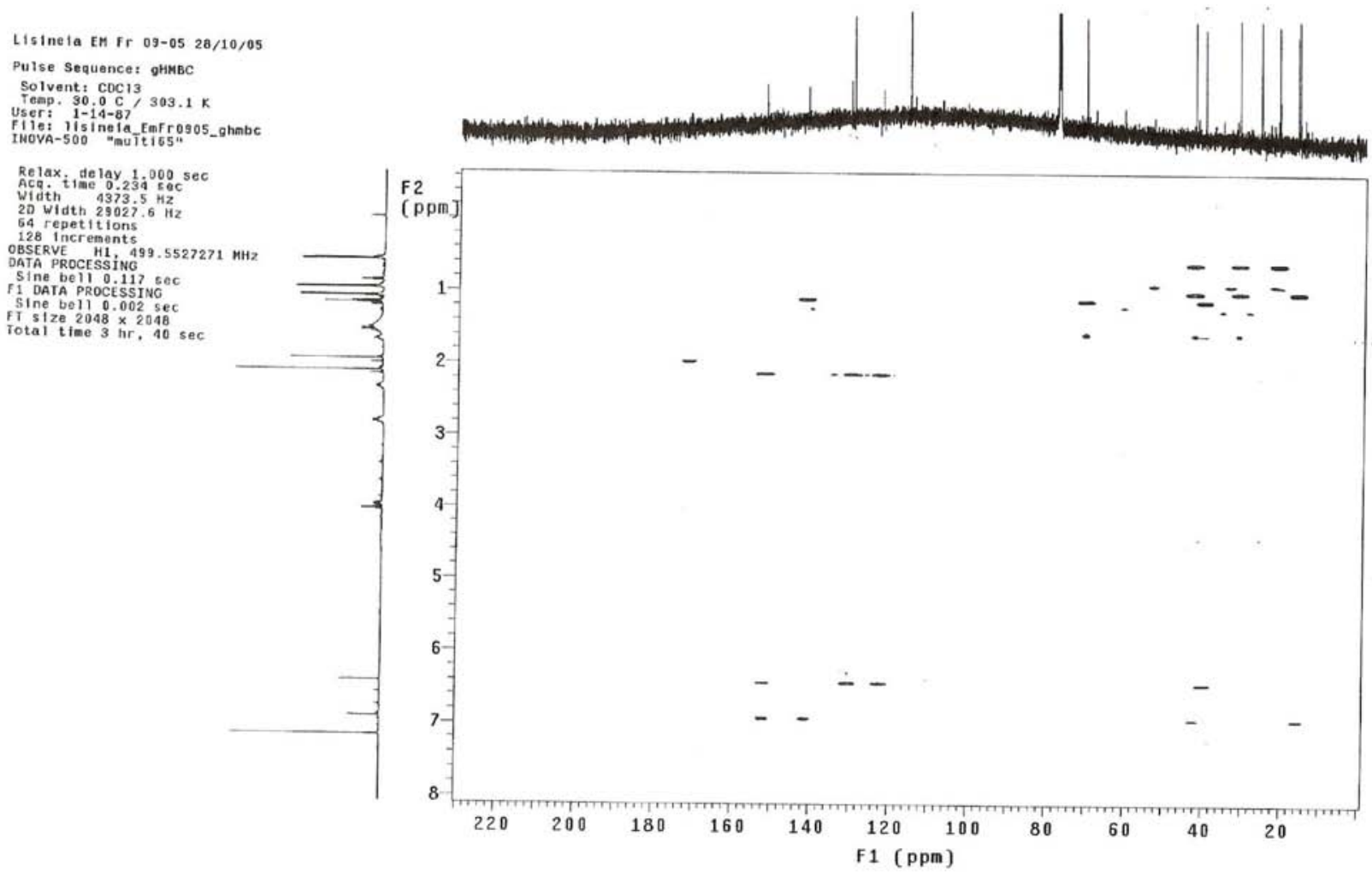

Figura 12S. Mapa de contorno de gHMBC de $2\left(\mathrm{CDCl}_{3}, 125 \mathrm{MHz}\right)$ 
Vol. 35, No. 11

Sesquiterpenos produzidos pelo fungo endofítico Phomopsis cassiae

S9

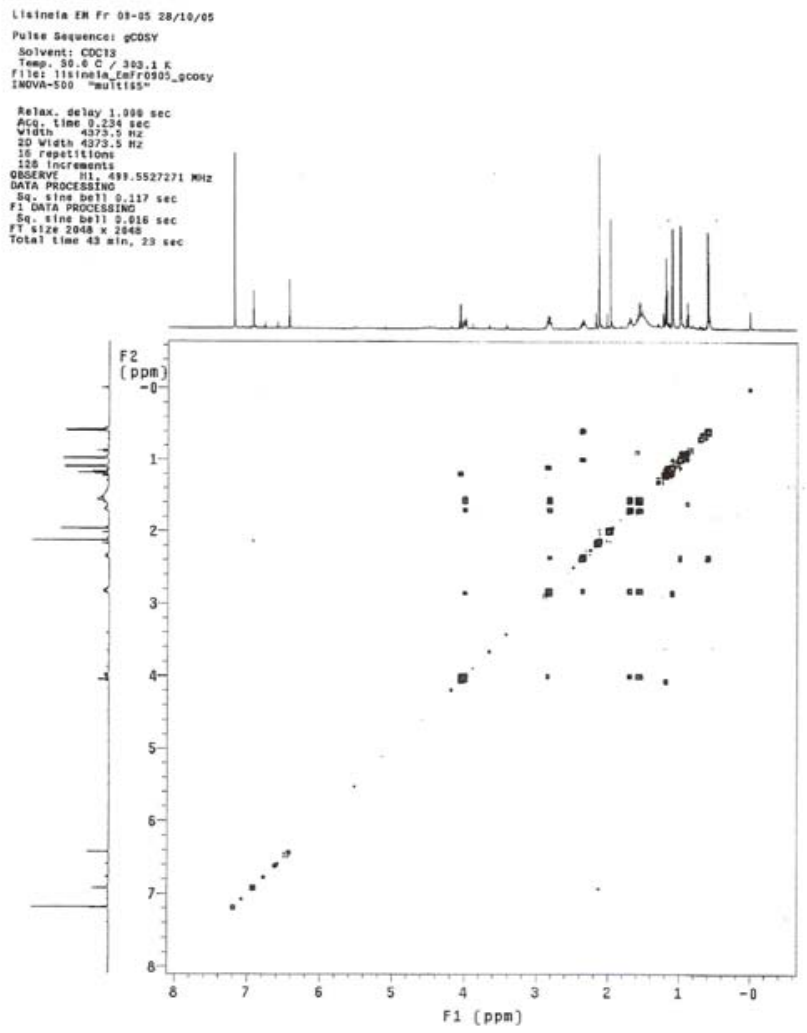

Figura 13S. Espectro de gCOSY de $2\left(\mathrm{CDCl}_{3}, 500 \mathrm{MHz}\right)$ 


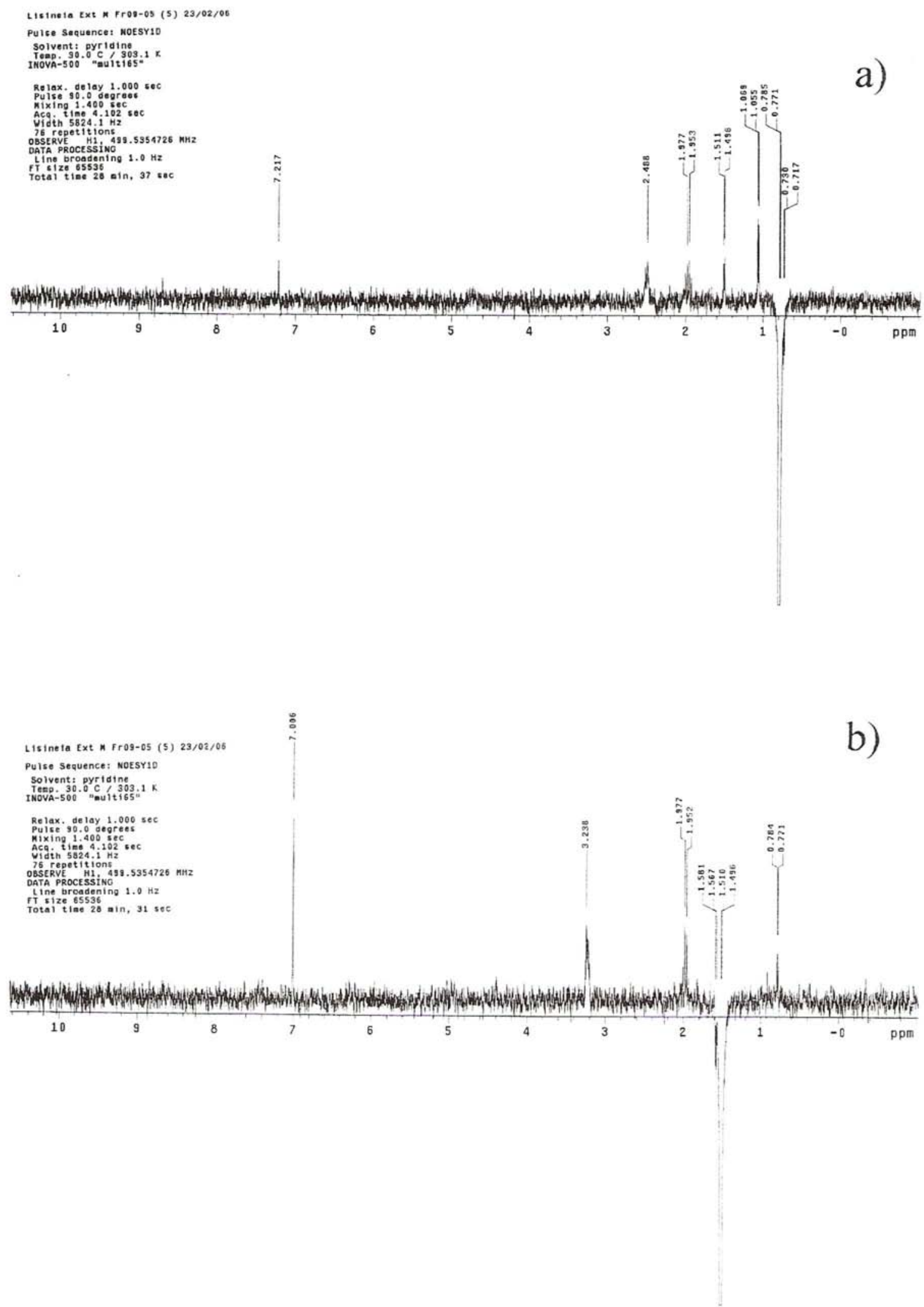

Figura 14S. Espectro de NOESY $1 D$ de $2\left(C_{5} D_{5} N, 500 \mathrm{MHz}\right)$, hidrogênios irradiados: a) $\left.H-13, \delta_{H} 0,75 ; b\right) H-14, \delta_{H} 1,54$ 


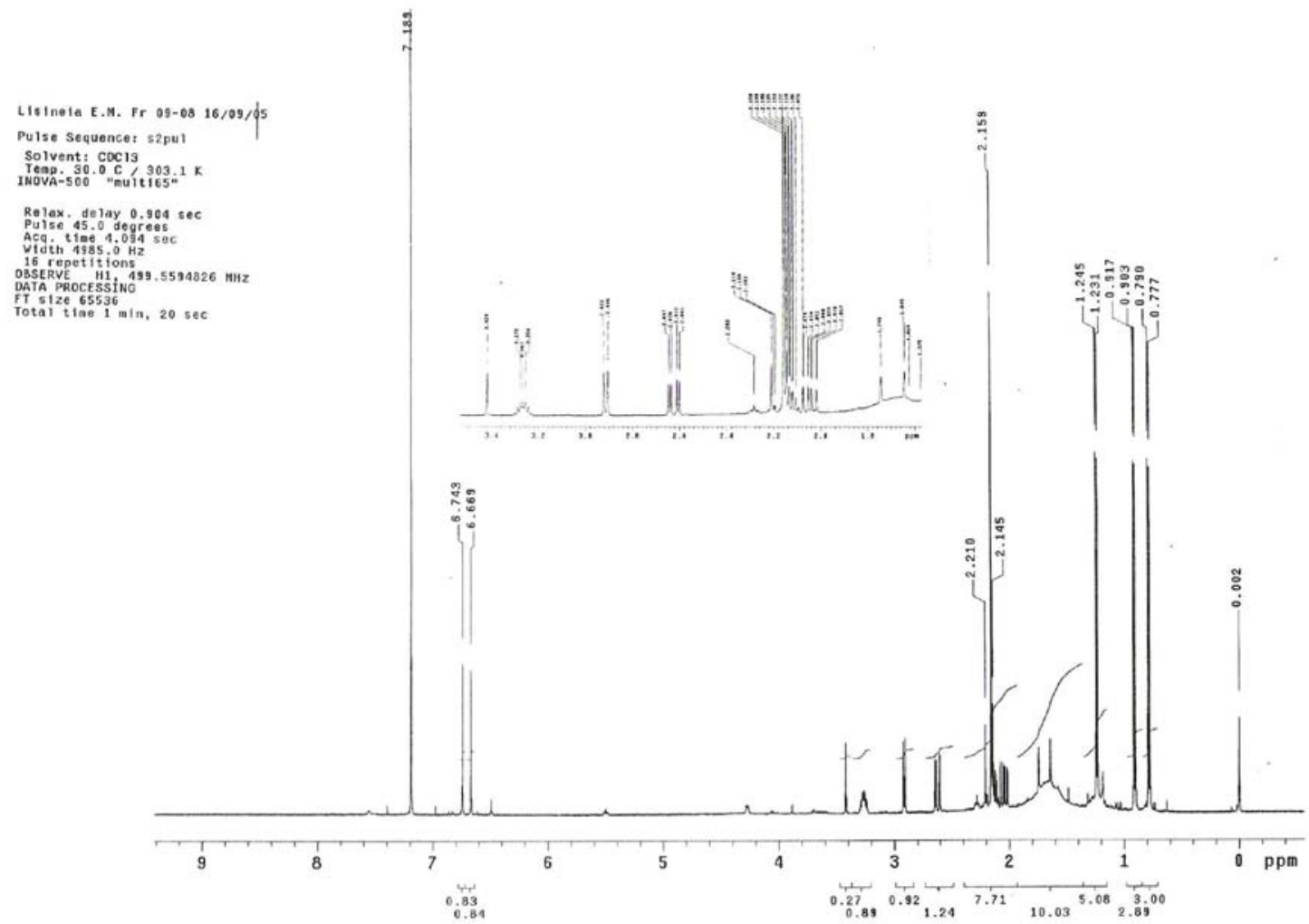

Figura 15S. Espectro de $\mathrm{RMN} \mathrm{de}{ }^{l} \mathrm{H}_{\mathrm{de}} \mathbf{3}\left(\mathrm{CDCl}_{3}, 500 \mathrm{MHz}\right)$

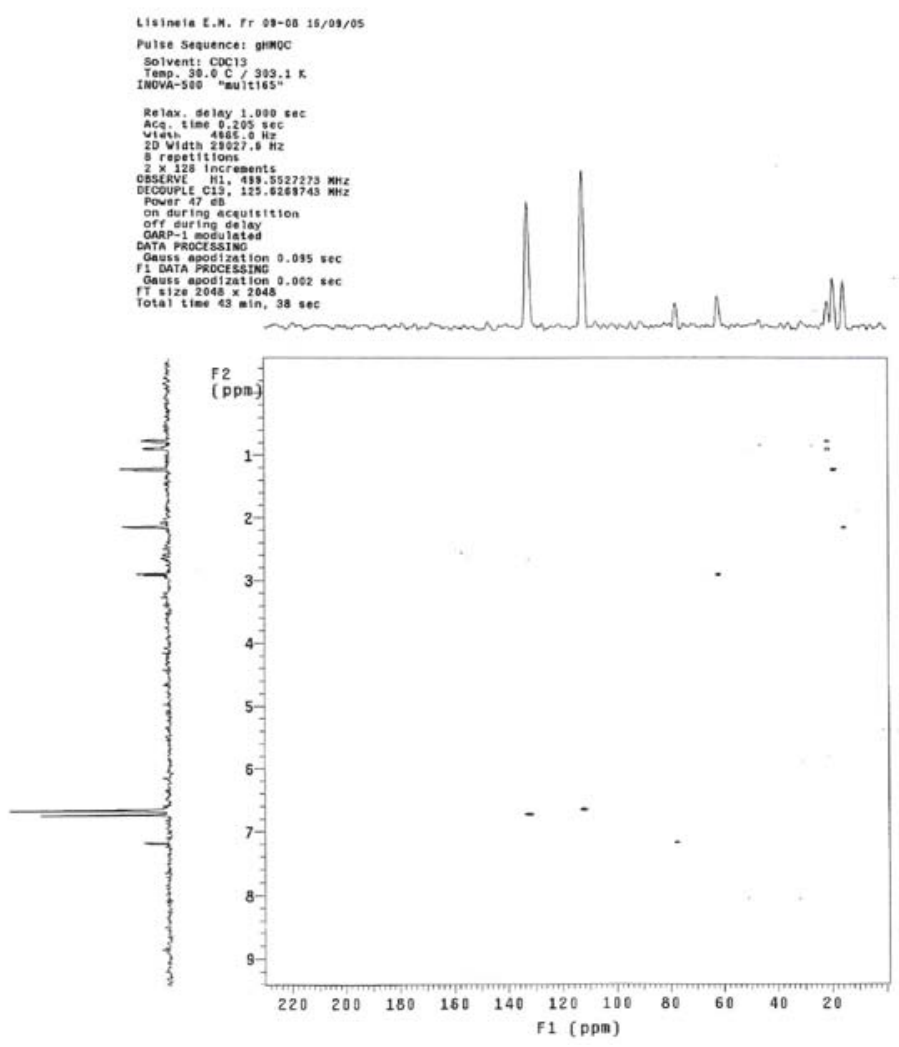

Figura 16S. Mapa de contorno de gHMQC de $3\left(\mathrm{CDCl}_{3}, 125 \mathrm{MHz}\right)$ 


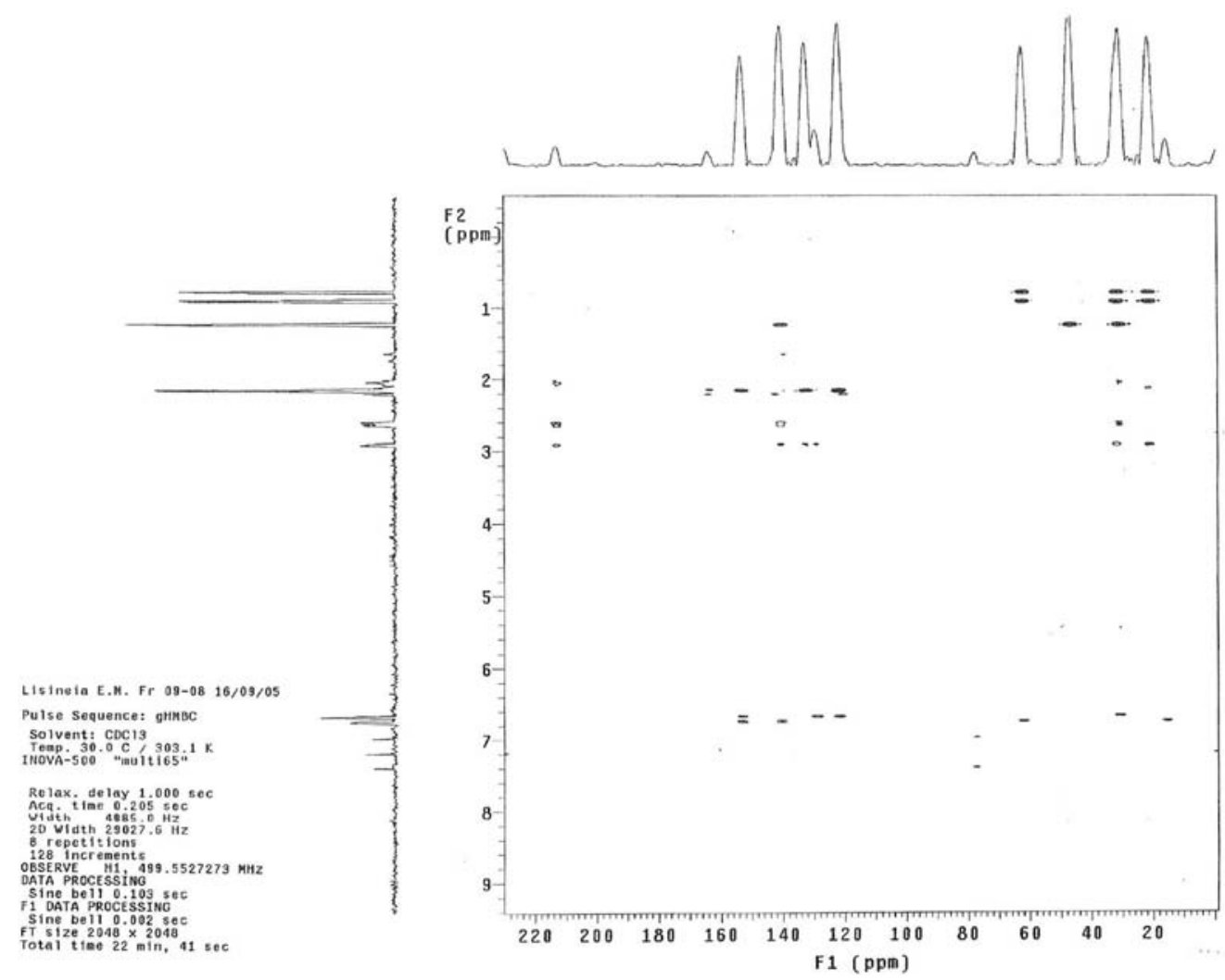

Figura 17S. Mapa de contorno de gHMBC de $3\left(\mathrm{CDCl}_{3}, 125 \mathrm{MHz}\right)$

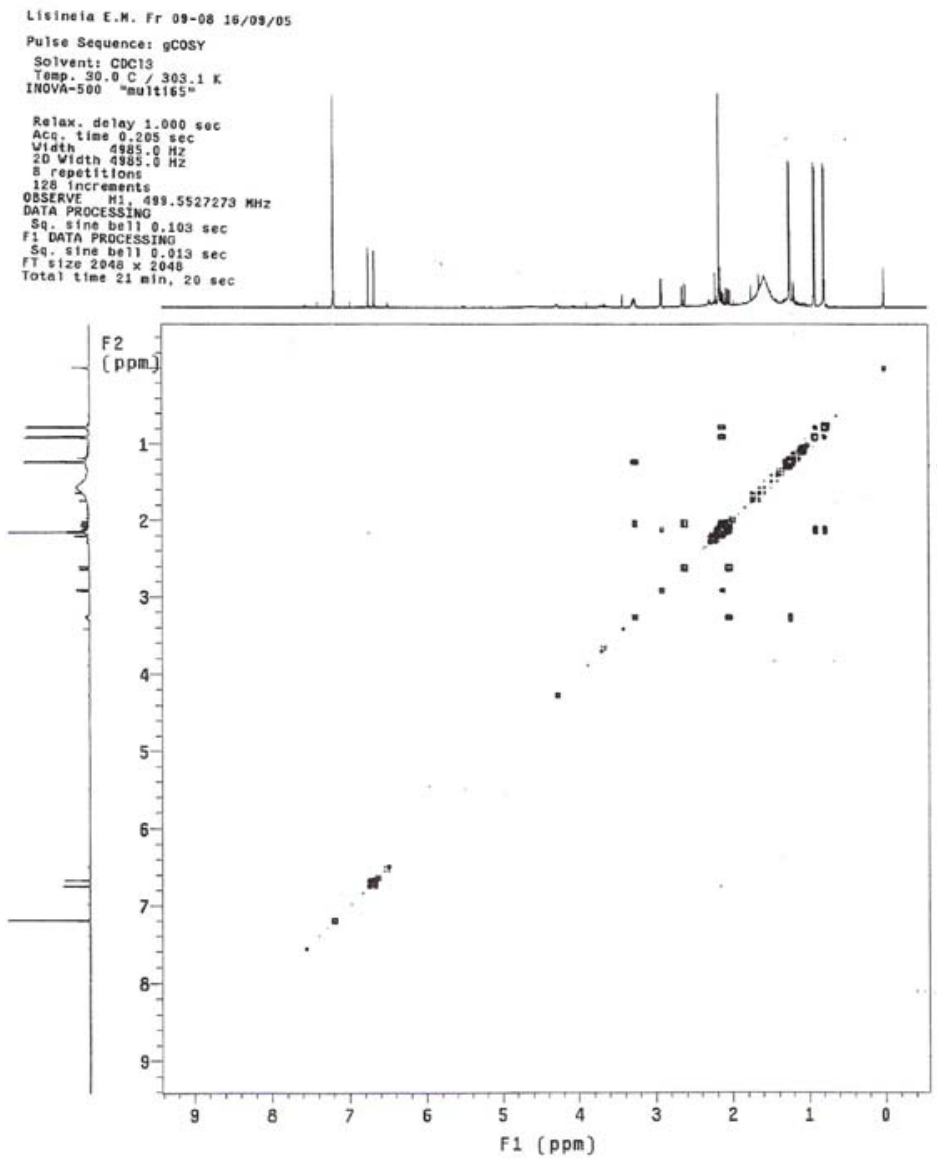

Figura 18S. Espectro de gCOSY de $\mathbf{3}\left(\mathrm{CDCl}_{3}, 500 \mathrm{MHz}\right)$ 


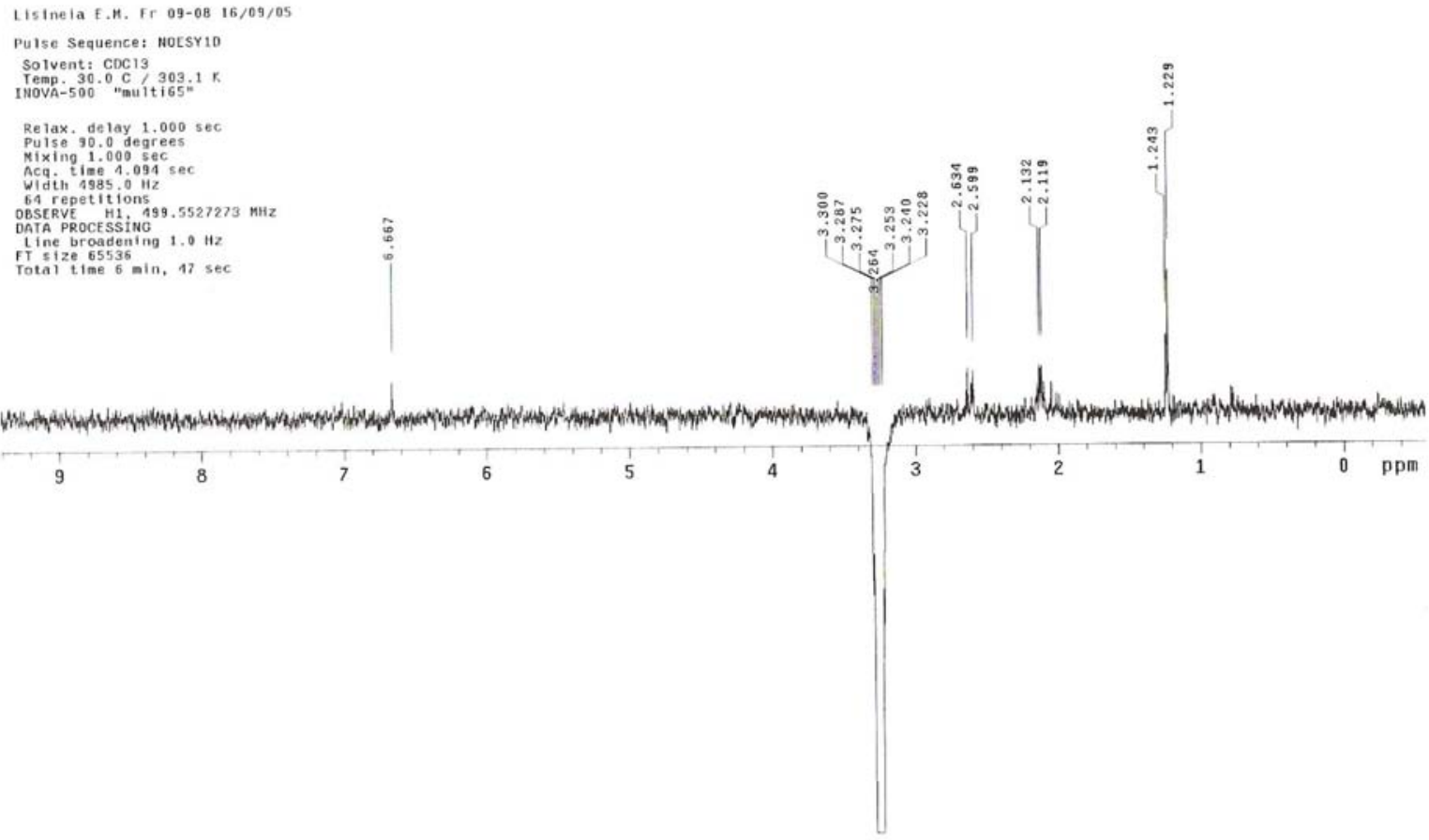

Figura 19S. Espectro de NOESY $1 D$ de $3\left(\mathrm{CDCl}_{3}, 500 \mathrm{MHz}\right)$, Irradiação do $\mathrm{H}-1, \delta_{H^{3}} 3,27$

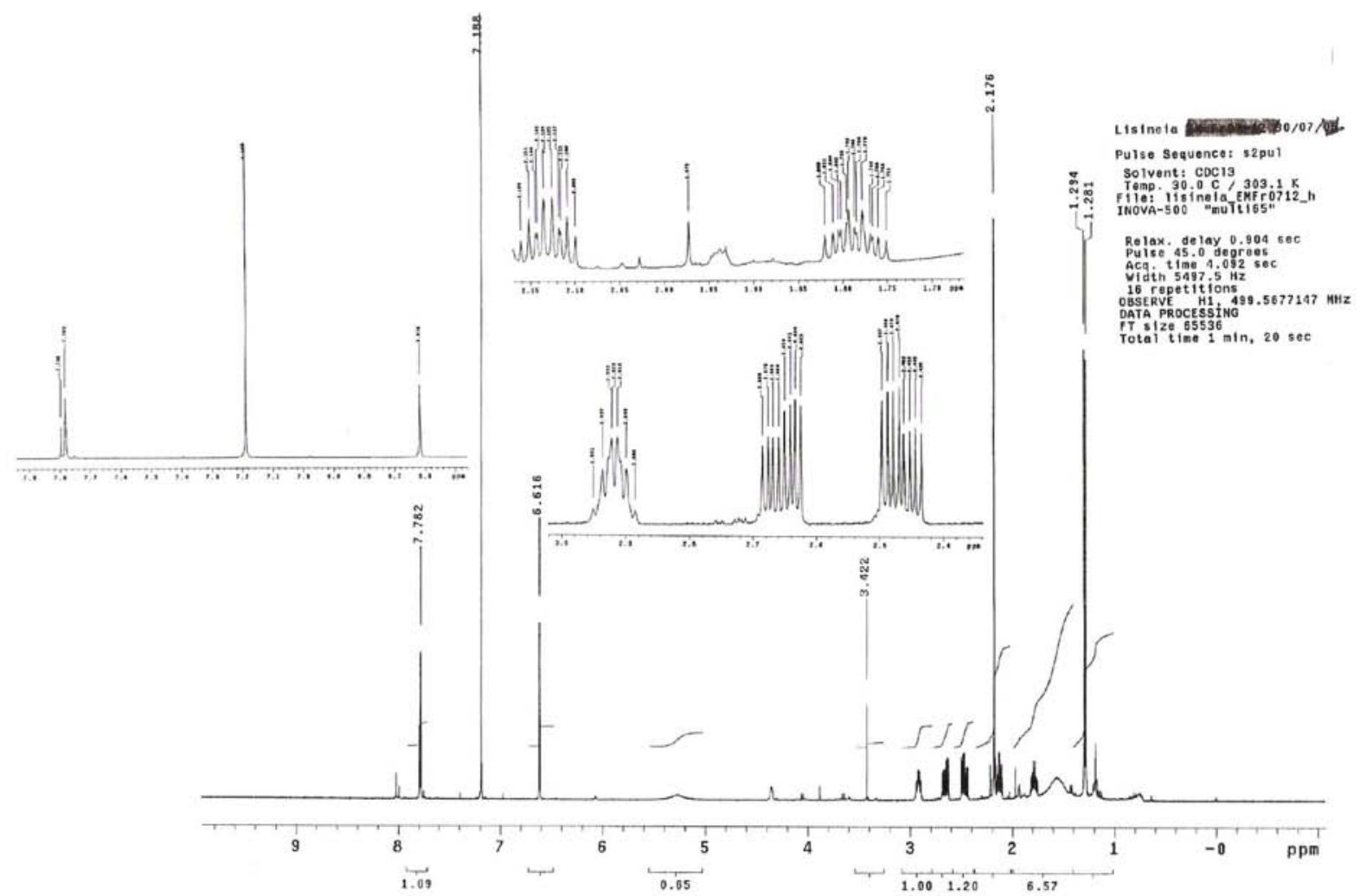

Figura 20S. Espectro de $\mathrm{RMN} \mathrm{de}{ }^{l} \mathrm{H}$ de $4\left(\mathrm{CDCl}_{3}, 500 \mathrm{MHz}\right)$ 

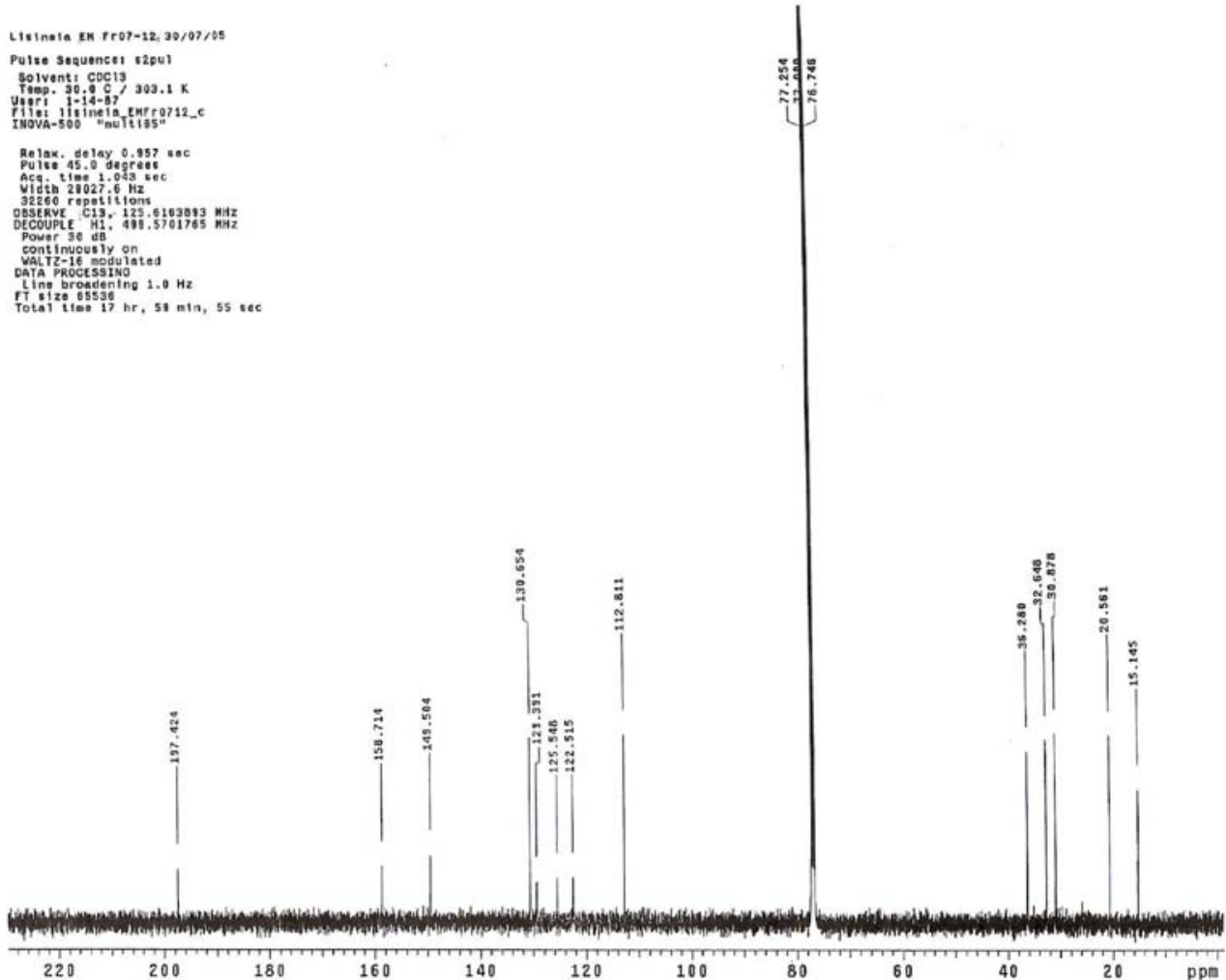

Figura 21S. Espectro de $\mathrm{RMN} d e{ }^{13} \mathrm{C}$ de $4\left(\mathrm{CDCl}_{3}, 125 \mathrm{MHz}\right)$

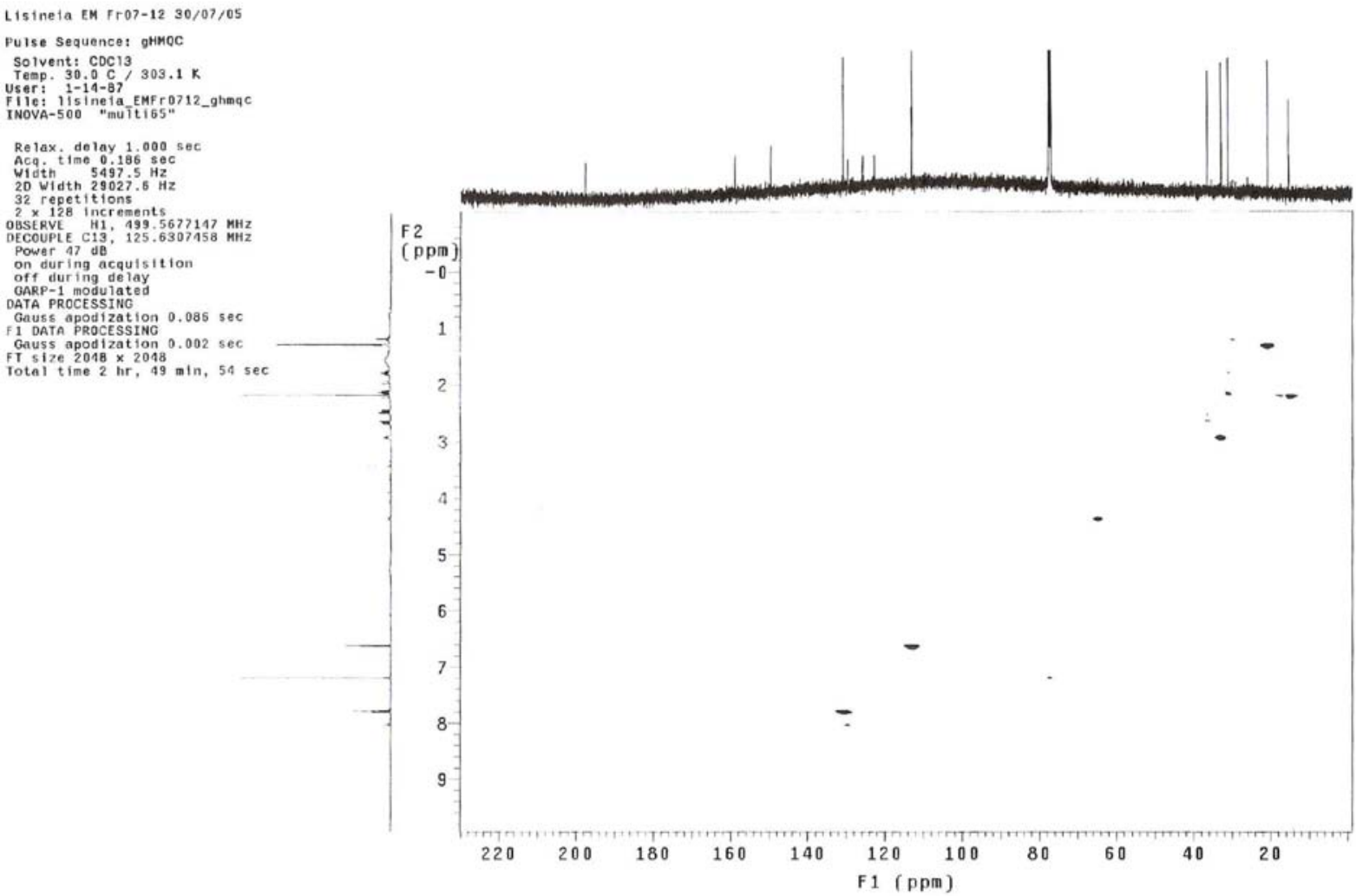

Figura 22S. Mapa de contorno de gHMQC de $4\left(\mathrm{CDCl}_{3}, 125 \mathrm{MHz}\right)$ 


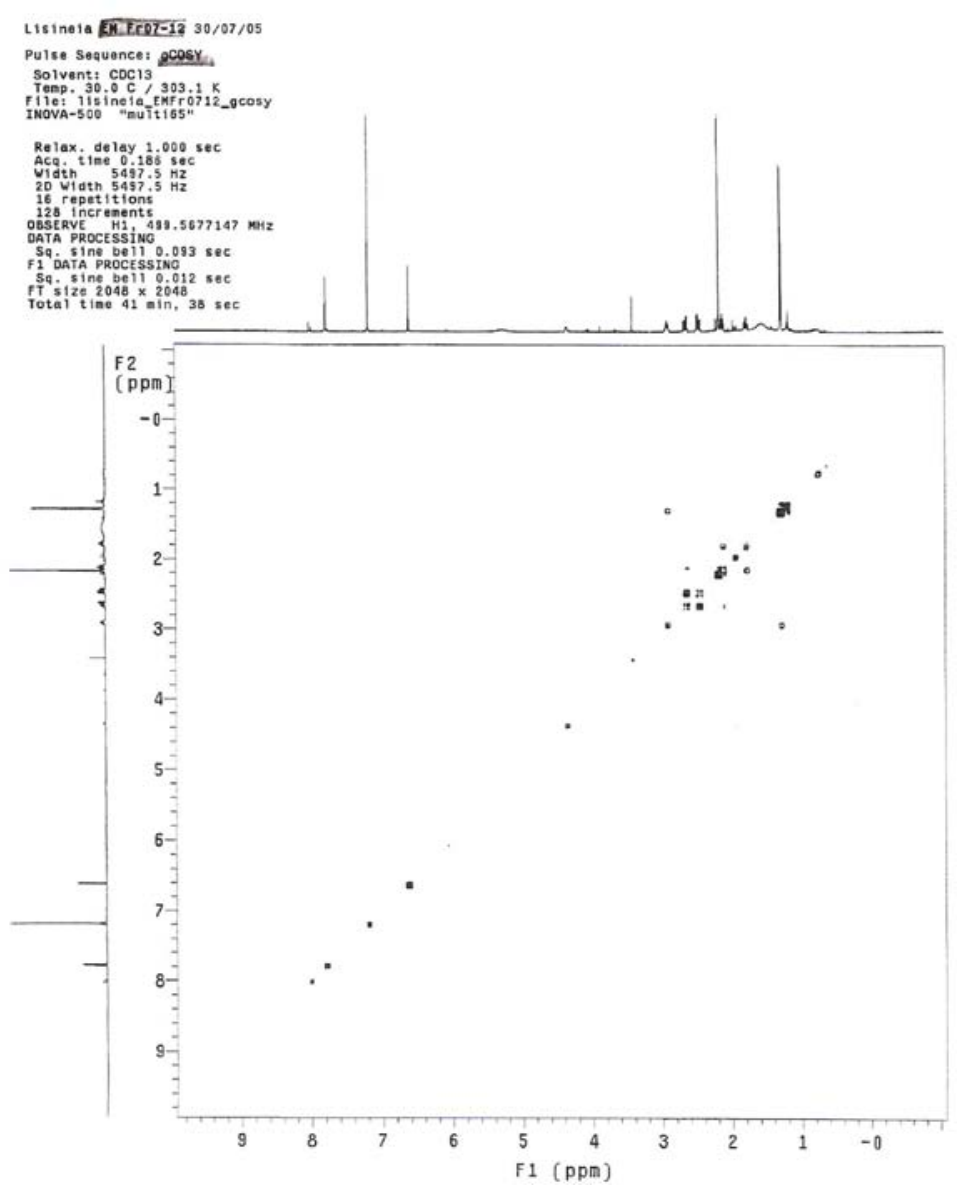

Figura 23S. Espectro de gCOSY de $4\left(\mathrm{CDCl}_{3}, 500 \mathrm{MHz}\right)$

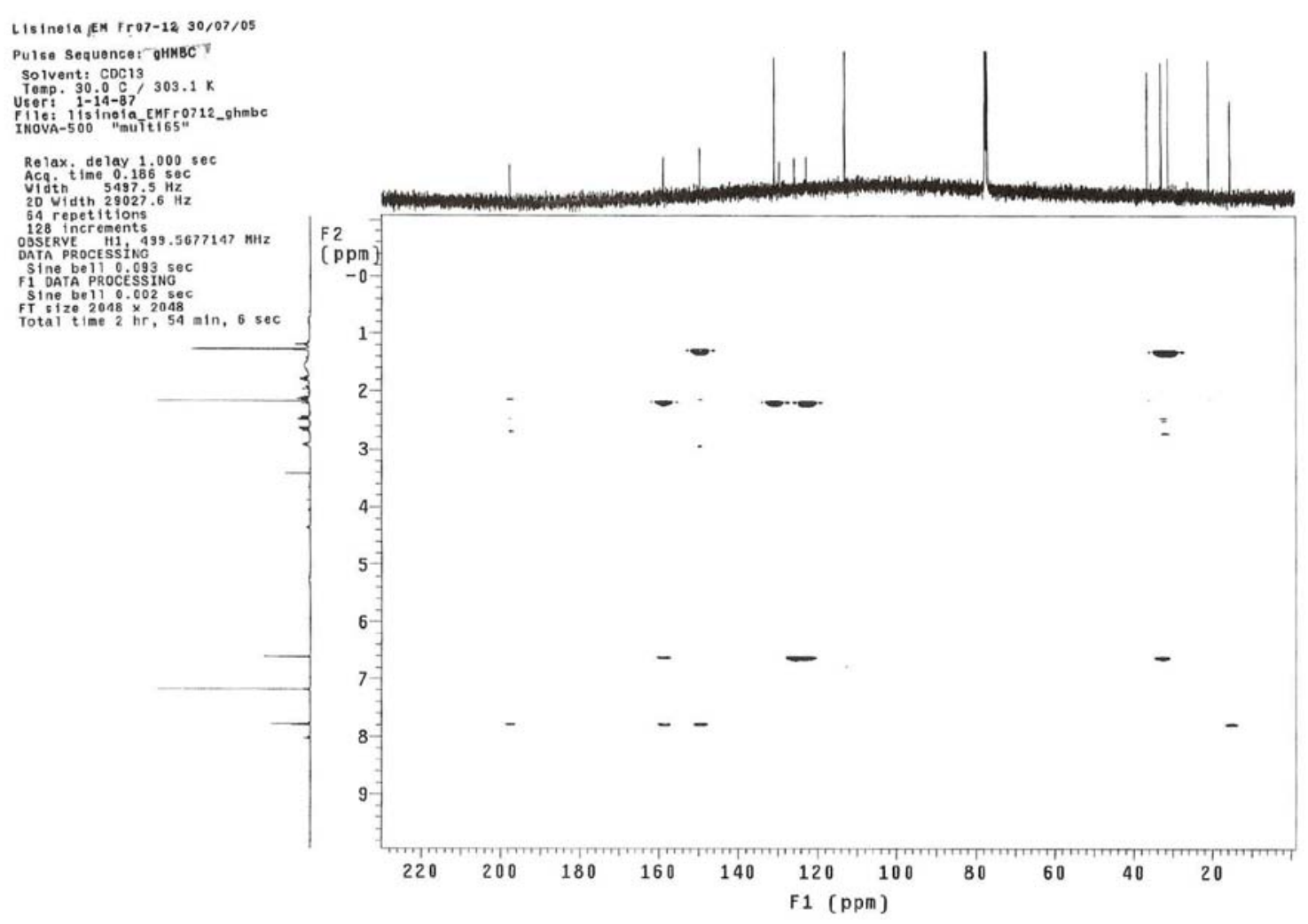

Figura 24S. Mapa de contorno de gHMBC de $4\left(\mathrm{CDCl}_{3}, 125 \mathrm{MHz}\right)$ 